\title{
Total synthesis and absolute configuration of avenolide, extracellular factor in Streptomyces avermitilis
}

\author{
Miho Uchida ${ }^{1}$, Satoshi Takamatsu ${ }^{2,4}$, Shiho Arima ${ }^{1}$, Kiyoko T Miyamoto ${ }^{3}$, Shigeru Kitani ${ }^{3}$, Takuya Nihira ${ }^{3}$, \\ Haruo Ikeda ${ }^{2}$ and Tohru Nagamitsu ${ }^{1}$ \\ The first total synthesis of extracellular factor, "Avenolide", in Streptomyces avermitilis has been achieved using a convergent \\ approach. The stereogenic centers in two key segments were installed using Sharpless epoxidation and dihydroxylation. This \\ synthetic study allowed the determination of the absolute configuration of avenolide as $4 S, 10 R$, and yielded important \\ information on its structure-activity relationship.
}

The Journal of Antibiotics (2011) 64, 781-787; doi:10.1038/ja.2011.90; published online 12 October 2011

Keywords: absolute configuration; avenolide; first total synthesis

\section{INTRODUCTION}

In several streptomycete microorganisms, $\gamma$-butyrolactones have important, sometimes crucial, roles as extracellular factors in determining the onset of secondary metabolite production and morphological differentiation. Up to date, the structures of all extracellular factors are consisted of $\gamma$-butyrolactones. ${ }^{1,2}$ A-factor is required for streptomycin biosynthesis and sporulation in Streptomyces griseus ${ }^{1,2}$ and virginia butanolide appears to control virginiamycin biosynthesis in Streptomyces virginiae. ${ }^{3-5}$ Other studies $\gamma$-butyrolactones include IM-2 elicits production of showdomycin and minimycin in Streptomyces lavendulae FRI-5 ${ }^{3-5}$ and SCB1 also elicits the precocious production of actinorhodin in Streptomyces coelicolor A3(2)..$^{3-5}$

Genome-sequenced Streptomyces avermitilis is an important industrial microorganism for the production of anthelmintic and insecticidal macrocyclic lactone, avermectin, which is used as antiparasitic agents in the medical, veterinary and agricultural fields. To identify the extracellular factor(s) controlling avermectin production in Streptomyces avermitilis, about $1 \mathrm{mg}$ of the extracellular factor from 10001 of culture filtrate was isolated and purified by chromatographic separation steps. ${ }^{6}$ Since spectroscopic analyses of the extracellular factor purified have elucidated that the factor is a new butenolide structure including two stereogenic centers (Scheme 1) but not $\gamma$ butyrolactone structure, the extracellular factor is named as "avenolide." The absolute configuration could not be elucidated, however, due to the trace quantities isolated and the presence of contaminants. Only the configuration of $\mathrm{C} 4$ ( $S$, avenolide numbering) was proposed, by comparison of the $\mathrm{CD}$ spectra of avenolide and similar butenolide compounds. ${ }^{7,8}$
To undertake further biochemical properties of avenolide, the determination of the absolute structure, access to an adequate supply of avenolide and the elucidation of the structure-activity relationship are required. We describe here the first total synthesis of avenolide, which represents significant progress towards these objectives.

\section{RESULTS AND DISCUSSION}

We first embarked on the synthesis of $(4 S, 10 R)$-avenolide (1). As outlined in Scheme 1, our retrosynthetic strategy is convergent. We speculated that 1 could be derived from epoxide 2 via ring closing metathesis, which itself could be constructed by the coupling of aldehyde 3 and iodide 4 . This route should provide a concise route amenable to large-scale synthesis of avenolide as well as various avenolide analogs, including stereoisomers.

The synthesis of $\mathbf{1}$ commenced with esterification of a commercially available $\beta$-methallyl alcohol with $p$-anisic acid (Scheme 2). Subsequent Sharpless dihydroxylation ${ }^{9,10}$ using (DHQ) ${ }_{2}$ PHAL as a chiral ligand afforded 5 (93\% ee). ${ }^{11-13}$ (The enantiomeric excess was determined by chiral HPLC analysis (condition: DAICEL CHIRALPAK AS-3 $(0.46 \mathrm{~cm} \phi \times 25 \mathrm{~cm}), 3: 1$ hexanes/2-propanol mobile phase (flow rate at $1.0 \mathrm{ml}$ per $\mathrm{min}$ ) and detection at $254 \mathrm{~nm}$ (room temperature, rt)). Tosylation of the primary alcohol followed by a substitution reaction with $\mathrm{Me}_{2} \mathrm{CuCNLi}_{2}$ and methanolysis gave diol 6. After acetal formation, DIBAL reduction and Parikh-Doering oxidation $^{14}$ furnished the desired aldehyde 3.

Another key intermediate 4 was derived from a commercially available 1,5-pentanediol. Mono-TBS protection, TEMPO oxidation, Wittig olefination with $\mathrm{Ph}_{3} \mathrm{P}=\mathrm{CHCO}_{2} \mathrm{Et}$ and DIBAL reduction

\footnotetext{
${ }^{1}$ School of Pharmacy, Kitasato University, Tokyo, Japan; ${ }^{2}$ Kitasato Institute for Life Sciences, Kitasato University, Kanagawa, Japan and ${ }^{3}$ International Center for Biotechnology, Osaka University, Osaka, Japan

${ }^{4}$ Current address: School of Pharmacy, Nihon University, 7-7-1 Narashinodai, Funabashi, Chiba 274-8555, Japan

Correspondence: Professor H Ikeda, Kitasato Institute for Life Sciences, Kitasato University, 1-15-1 Kitasato, Minami-ku, Sagamihara, Kanagawa 252-0373, Japan.

E-mail: ikeda@Is.kitasato-u.ac.jp

or Professor T Nagamitsu, School of Pharmacy, Kitasato University, 5-9-1 Shirokane, Minato-ku, Tokyo 108-8641, Japan.

E-mail: nagamitsut@pharm.kitasato-u.ac.jp
}

Received 27 June 2011; revised 21 August 2011; accepted 30 August 2011; published online 12 October 2011 


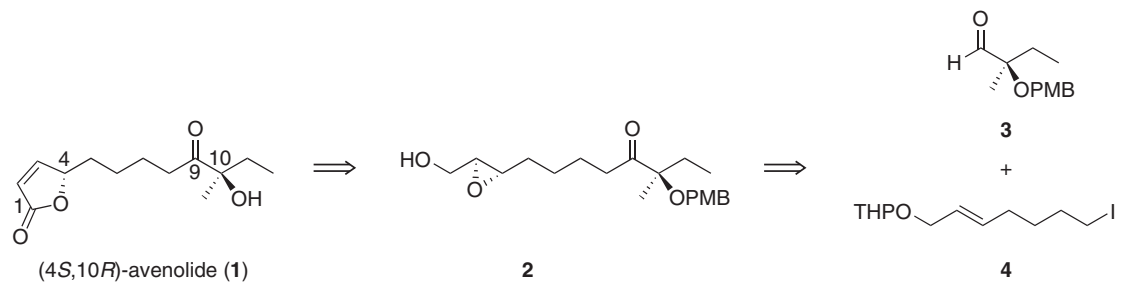

Scheme 1 Retrosynthetic analysis of $(4 S, 10 R)$-avenolide (1).
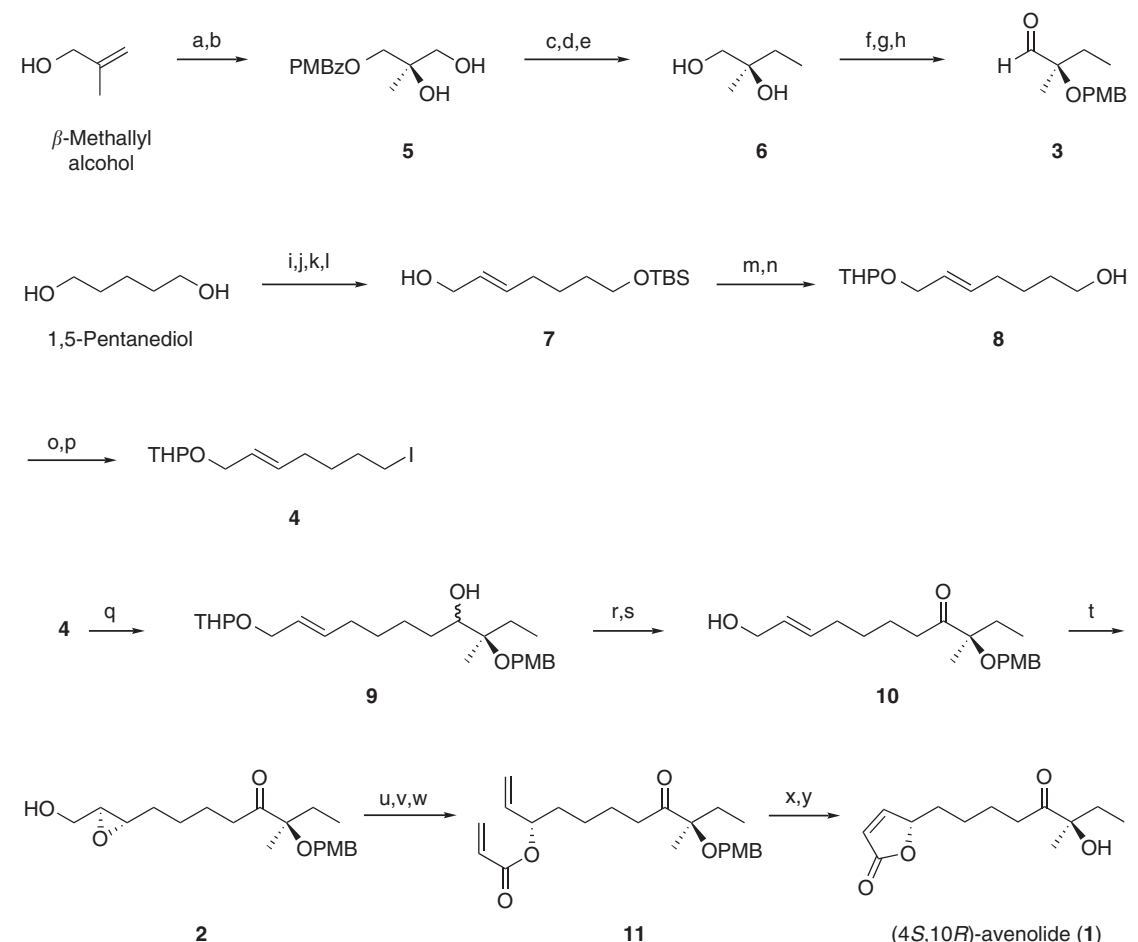

Scheme 2 Reagents and conditions: (a) p-anisic acid, WSC, DMAP, $\mathrm{CH}_{2} \mathrm{Cl}_{2}$, rt, $99 \%$; (b) $\mathrm{K}_{3} \mathrm{Fe}(\mathrm{CN})_{6}, \mathrm{~K}_{2} \mathrm{CO}_{3},(\mathrm{DHQ})_{2} \mathrm{PHAL}, \mathrm{K}_{2} \mathrm{OsO}{ }_{4}(\mathrm{OH})_{4}, t-\mathrm{BuOH}: \mathrm{H}_{2} \mathrm{O}=1: 1,0{ }^{\circ} \mathrm{C}$, quant., 93\% ee; (c) $p$ - $\mathrm{TsCl}, \mathrm{Et}_{3} \mathrm{~N}, \mathrm{Me}_{3} \mathrm{~N} \cdot \mathrm{HCl}, \mathrm{CH}_{2} \mathrm{Cl}_{2}$, rt, $99 \%$; (d) $\mathrm{CuCN}, \mathrm{MeLi}, \mathrm{THF}, 0{ }^{\circ} \mathrm{C}$; (e) $\mathrm{K}_{2} \mathrm{CO}_{3}, \mathrm{MeOH}, \mathrm{rt}, 90 \%$ (two steps); (f) p-methoxybenzaldehyde dimethylacetal, PPTS, rt, $\mathrm{CH}_{2} \mathrm{Cl}_{2}$, quant.; (g) DIBAL, $\mathrm{CH}_{2} \mathrm{Cl}_{2}, 0^{\circ} \mathrm{C}, 87 \%$; (h) $\mathrm{SO}_{3}$. Py, DMSO, $\mathrm{Et}_{3} \mathrm{~N}, \mathrm{CH}_{2} \mathrm{Cl}_{2}$, rt, $89 \%$; (i) $\mathrm{NaH}, \mathrm{TBSCl}, \mathrm{THF}, \mathrm{rt}, 80 \%$; (j) $\mathrm{Phl}(\mathrm{OAc})_{2}$, TEMPO, $\mathrm{CH}_{2} \mathrm{Cl}_{2}$, rt, quant.; (k) $\mathrm{Ph}_{3} \mathrm{P}=\mathrm{CHCO}_{2} \mathrm{Et}$, benzene, $80^{\circ} \mathrm{C}$, quant.; (I) DIBAL, $\mathrm{CH}_{2} \mathrm{Cl}_{2}, 0^{\circ} \mathrm{C}$, quant.; (m) PPTS, DHP, $\mathrm{CH}_{2} \mathrm{Cl}_{2}, 0^{\circ} \mathrm{C}$, quant.; (n) TBAF, THF, rt, quant.; (o) $\mathrm{MsCl}, \mathrm{Et}_{3} \mathrm{~N}, \mathrm{Me}_{3} \mathrm{~N} \cdot \mathrm{HCl}, \mathrm{CH}_{2} \mathrm{Cl}_{2}$, rt, $93 \%$; (p) Nal, acetone, reflux, quant.; (q) $t$-BuLi, pentane, $\mathrm{Et}_{2} \mathrm{O},-78^{\circ} \mathrm{C}$, then $3,-78{ }^{\circ} \mathrm{C}$ to $0{ }^{\circ} \mathrm{C}, 96 \%$; (r) TPAP, NMO, $\mathrm{CH}_{2} \mathrm{Cl}_{2}, \mathrm{rt}, 97 \%$; (s) PPTS, $\mathrm{MeOH}, \mathrm{rt}, 99 \%$; (t) (+)-DET, Ti(Oi-Pr) 4 , $4 \AA$ molecular sieves, $t-\mathrm{BuOOH}_{2} \mathrm{CH}_{2} \mathrm{Cl}_{2},-20{ }^{\circ} \mathrm{C}, 86 \%$; (u) $\mathrm{PPh}, \mathrm{I}_{2}$, imidazole, THF:MeCN=4:1, rt, $80 \%$; (v) $\mathrm{Nal}, \mathrm{Zn}, \mathrm{MeOH}, 90^{\circ} \mathrm{C}$, quant.; (w) acryloyl chloride, DMAP, $\mathrm{Et}_{3} \mathrm{~N}, \mathrm{CH}_{2} \mathrm{Cl}_{2}, \mathrm{rt}, 90 \%$; (x) $\mathrm{DDQ}, \mathrm{CH}_{2} \mathrm{Cl}_{2}: \mathrm{H}_{2} \mathrm{O}=2: 1, \mathrm{rt}$, quant.; (y) Grubbs second-generation catalyst, $\mathrm{CH}_{2} \mathrm{Cl}_{2}, 40{ }^{\circ} \mathrm{C}$, quant.

afforded E-allyl alcohol $7.15,16$ This was then converted to 8 by a two-step sequence of protecting group manipulations. Mesylation of the primary alcohol of $\mathbf{8}$ followed by treatment with sodium iodide gave rise to 4 .

The aldehyde 3 was coupled with an alkyl lithium species derived from halogen-lithium exchange of iodide $\mathbf{4}$ with $t$-BuLi to furnish alcohol $\mathbf{9}$ as a diastereomeric mixture. TPAP oxidation and THP deprotection under acidic conditions afforded ketoalcohol 10. Subsequent Sharpless asymmetric epoxidation ${ }^{17}$ using (+)-DET as a chiral ligand gave the desired epoxyalcohol 2. Transformation of the primary alcohol to iodide followed by treatment with zinc yielded the corresponding allyl alcohol, which was acylated with acryloyl chloride to provide 11. Finally, deprotection of the $\mathrm{PMB}$ group by treatment with DDQ and ring closing metathesis ${ }^{18-22}$ using Grubbs second-generation catalyst afforded $\mathbf{1}$.

To determine the absolute configuration of $\mathbf{1}$, we also synthesized $(4 S, 10 S)$-avenolide (19), according to Scheme 2, with (DHQD) ${ }_{2}$ PHAL used instead of (DHQ) $)_{2}$ PHAL as the chiral ligand in Sharpless asymmetric dihydroxylation (Scheme 3 ).

We next analyzed the synthetic $\mathbf{1}$ and $\mathbf{1 9}$ with a chiral HPLC column (conditions: DAICEL CHIRALPAK IA-3 $(0.46 \mathrm{~cm} \phi \times 25 \mathrm{~cm}), \mathrm{EtOH}$ mobile phase (flow rate of $0.3 \mathrm{ml}$ per $\mathrm{min}$ ) and detection at $200 \mathrm{~nm}$ $\left.\left(0{ }^{\circ} \mathrm{C}\right)\right)$. When a 1:1 mixture of $\mathbf{1}$ and $\mathbf{1 9}$ was injected onto the chiral HPLC system, the two diastereomers were completely resolved, as shown in Figure 1a. Figures $1 \mathrm{~b}$ and $\mathrm{c}$ show the HPLC chromatogram of 1 and 19, respectively. An authentic sample of the natural avenolide was also analyzed by HPLC, and its retention time was identical to that of 1 as shown in Figure 1d. This result establishes the absolute configuration of avenolide as $4 S, 10 R$.

We also synthesized $(4 R, 10 R)$-avenolide (22) and 10-deoxy avenolide (28) for structure-activity relationship studies as shown in Scheme 4. (The spectrum data of compounds in Schemes 3 and 4 are provided as Supplementary information.) The synthesis of $\mathbf{2 2}$ was achieved 

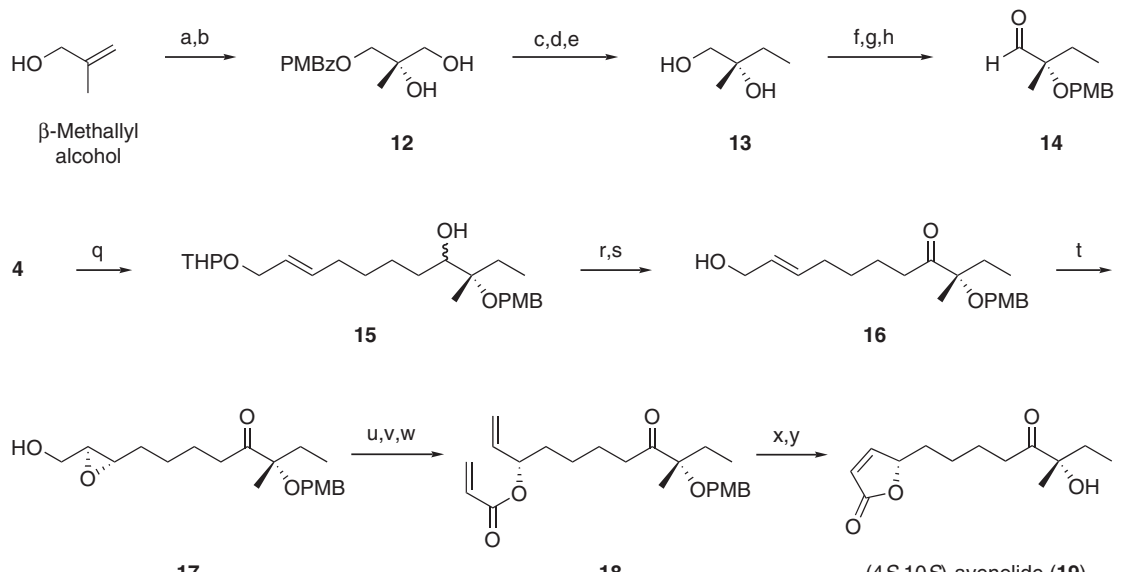

Scheme 3 Reagents and conditions: the same reaction conditions as those described in Scheme 2 were used, unless otherwise noted. (b) $\mathrm{K}_{3} \mathrm{Fe}(\mathrm{CN})_{6}$, $\mathrm{K}_{2} \mathrm{CO}_{3},(\mathrm{DHQD})_{2} \mathrm{PHAL}, \mathrm{K}_{2} \mathrm{OsO}_{4}(\mathrm{OH})_{4}, t-\mathrm{BuOH}: \mathrm{H}_{2} \mathrm{O}=1: 1,0{ }^{\circ} \mathrm{C}$, quant., $93 \%$ ee ${ }^{11-13}$; (q) $t$-BuLi, pentane, $\mathrm{Et}_{2} \mathrm{O},-78^{\circ} \mathrm{C}$, then $14,-78{ }^{\circ} \mathrm{C}$ to $0^{\circ} \mathrm{C}, 88 \%$.
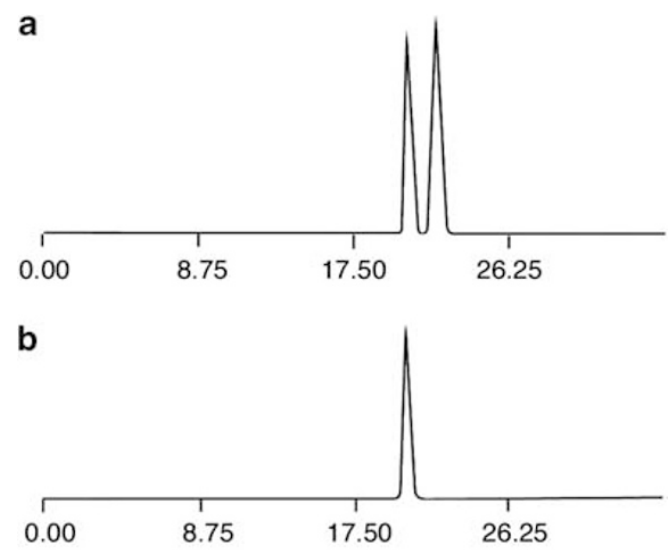

C

$\begin{array}{llcc}1 & 1 & 1 \\ 0.00 & 8.75 & 17.50 & 26.25\end{array}$

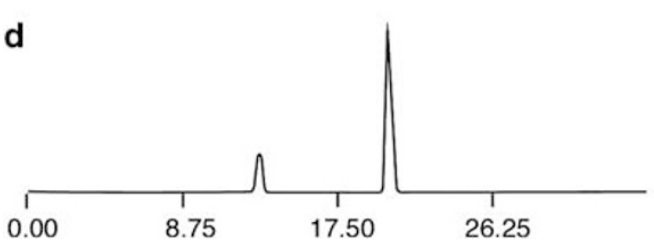

Figure 1 HPLC chromatograms of natural and synthetic samples of avenolide (1 and 19). The abscissa axis indicates retention time ( $\mathrm{min})$. (a) $1: 1$ mixture of 1 and 19 ; (b) $\mathbf{1}$; (c) 19 ; (d) natural avenolide.

according to our synthetic procedure for $\mathbf{1}$ as shown in Scheme 2, but using (-)-DET as the chiral ligand during Sharpless epoxidation. In contrast, the synthesis of $\mathbf{2 8}$ required a slightly altered synthetic route. A commercially available (S)-2-methyl-1-butanol was subjected to TEMPO oxidation and dithioacetalization to afford $23,{ }^{23}$ which was coupled with iodide 4 to give 24. THP deprotection followed by treatment with [bis(trifluoroacetoxy)iodo]benzene furnished ketone 25. The conversion of $\mathbf{2 5}$ into 10-deoxy avenolide (28) was carried out following the same synthetic procedure as that developed for the synthesis of 1 shown in Scheme 2.

Synthetic 1 demonstrated identical activity to that of natural 1 . In contrast, the rAvaR1-ligand activities of the C10-epimer (19), the C4-epimer (22) and the 10-deoxy avenolide (28) demonstrated only one-half, one twenty-fifth and one hundredth of the activity of the natural product, respectively. ${ }^{6}$ It can therefore be concluded that the stereochemistry at $\mathrm{C} 4$ and $\mathrm{C} 10$, and the presence of the hydroxyl group at $\mathrm{C} 10$, are important to the activity of avenolide as a rAvaR1 ligand.

In conclusion, we have achieved the total synthesis of avenolide and determined its absolute configuration. By extending the synthetic methodology, we have generated a number of structural analogs, which provide information on the structure-activity relationship of avenolide. Further biological studies on the synthetic avenolide are currently underway and will be reported in due course.

\section{EXPERIMENTAL PROCEDURE}

\section{General}

IR spectra were obtained using a Horiba FT-710 spectrophotometer (Horiba, Kyoto, Japan). ${ }^{1} \mathrm{H}$ and ${ }^{13} \mathrm{C}-\mathrm{NMR}$ spectra were obtained on Mercury-300 and UNITY-400 spectrometers (Agilent Technologies, Santa Clara, CA, USA), and chemical shifts are reported on the $\delta$ scale, using TMS as an internal reference. MS spectra were measured on JEOL JMS-700, JEOL JMS-T-100LP and JEOL JMSAX505HA spectrometers. Optical rotations were recorded on a JASCO DIP-1000 polarimeter (Jasco, Hachioji, Japan). Commercial reagents were used without further purification unless otherwise indicated. Organic solvents were distilled and dried over molecular sieves ( 3 or $4 \AA$ ). Reactions were performed in flame-dried glassware under positive Ar pressure while stirring with a magnetic stirrer bar unless otherwise indicated. Flash chromatography was performed on silica gel $60 \mathrm{~N}$ (spherical, neutral, particle size $40-50 \mathrm{~mm}$ ). TLC was performed on $0.25 \mathrm{~mm}$ Merck silica gel 60 F254 plates (Merck, Darmstadt, Germany) and visualized by UV $(254 \mathrm{~nm})$, and using phosphomolybdic acid and p-anisaldehyde as TLC stains.

(R)-2,3-Dihydroxy-2-methylpropyl-4-methoxybenzoate (5). To a solution of $\beta$-methallyl alcohol $(3.50 \mathrm{ml}, 41.3 \mathrm{mmol})$ in $\mathrm{CH}_{2} \mathrm{Cl}_{2}(83 \mathrm{ml})$ were added DMAP (504 mg, $4.13 \mathrm{mmol}$ ), $N$-(3-dimethylaminopropyl)- $N^{\prime}$-ethylcarbodiimide hydrochloride (WSC, $8.68 \mathrm{~g}, 45.4 \mathrm{mmol}$ ) and $p$-anisic acid $(6.91 \mathrm{~g}, 45.5 \mathrm{mmol}$ ) at $\mathrm{rt}$ under $\mathrm{N}_{2}$. The reaction mixture was stirred for $2.5 \mathrm{~h}$ at $\mathrm{rt}$. The reaction was quenched with $\mathrm{H}_{2} \mathrm{O}$ and the aqueous phase was extracted with $\mathrm{CH}_{2} \mathrm{Cl}_{2}$. The combined organic extracts were dried over anhydrous $\mathrm{Na}_{2} \mathrm{SO}_{4}$ and concentrated in vacuo. The residue was purified by flash column chromatography (30:1 hexanes/EtOAc) to furnish the corresponding $p$-methoxybenzoate $(8.38 \mathrm{~g}, 99 \%)$ as a colorless oil. 


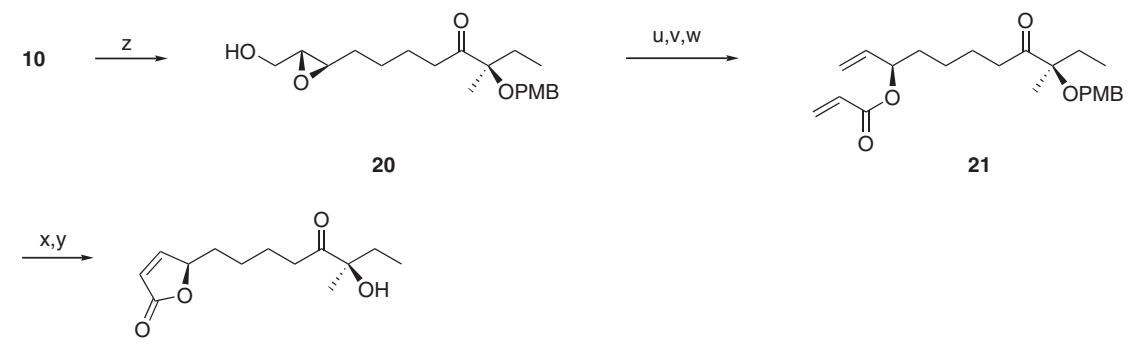

$(4 R, 10 R)$-avenolide (22)

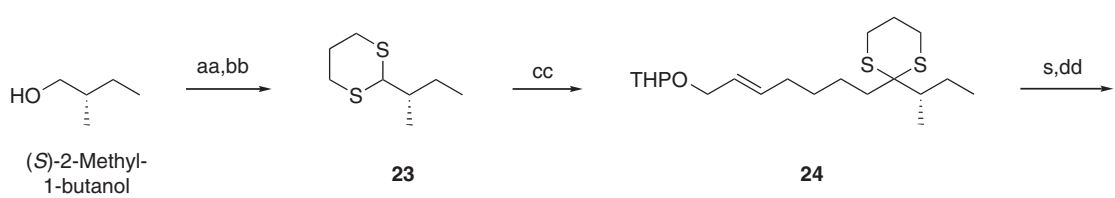<smiles>C=C[C@H](CCCCC(=O)[C@H](C)CC)OC(=O)/C=C/C#N</smiles><smiles>CC[C@H](C)C(=O)CCCCC[C@H](C)CC</smiles>

Scheme 4 Reagents and conditions: the same reaction conditions as those described in Scheme 2 were used unless otherwise noted. (z) $(-)$-DET, Ti(Oi-Pr) 4 , $4 \AA$ molecular sieves, $t$ - $\mathrm{BuOOH}, \mathrm{CH}_{2} \mathrm{Cl}_{2},-20^{\circ} \mathrm{C}, 70 \%$; (aa) PhI(OAc) $)_{2}, \mathrm{TEMPO}, \mathrm{CH}_{2} \mathrm{Cl}_{2}$, rt; (bb) 1,3-pentanedithiol, $\mathrm{BF}_{3} \cdot \mathrm{Et}_{2} \mathrm{O},-5^{\circ} \mathrm{C}, 70 \%$ (two steps); (cc) $t$-BuLi, THF:HMPA $=10: 1,-78{ }^{\circ} \mathrm{C}$, then 4, 82\%; (dd) [bis(trifluoroacetoxy)iodo]benzene, $\mathrm{CH}_{3} \mathrm{CN}: \mathrm{H}_{2} \mathrm{O}=9: 1$, quant.

IR $(\mathrm{KBr}) 3082,2939,2842,1715,1607,1511,1457 \mathrm{~cm}^{-1} ;{ }^{1} \mathrm{H}-\mathrm{NMR}(400 \mathrm{MHz}$, $\left.\mathrm{CDCl}_{3}\right) \delta 8.03(\mathrm{~d}, J=9.0 \mathrm{~Hz}, 2 \mathrm{H}), 6.92(\mathrm{~d}, J=9.0 \mathrm{~Hz}, 2 \mathrm{H}), 5.06$ (brs, $\left.1 \mathrm{H}\right), 4.97$ (brs, $1 \mathrm{H}), 4.72$ (brs, $2 \mathrm{H}), 3.85$ (s, 3H), 1.83 (brs, 3H); ${ }^{13} \mathrm{C}-\mathrm{NMR}(100 \mathrm{MHz}$, $\left.\mathrm{CDCl}_{3}\right) \delta 166.0,163.4,140.2,131.7,122.6,113.7,112.7,67.8,55.4,19.6$; HRMS (ESI', TFA-Na) calcd for $\mathrm{C}_{12} \mathrm{H}_{14} \mathrm{NaO}_{3} 229.0841[\mathrm{M}+\mathrm{Na}]^{+}$, found $\mathrm{m} / z 229.0841$.

To a solution of the $p$-methoxybenzoate $(4.00 \mathrm{~g}, 19.4 \mathrm{mmol})$ in $t-\mathrm{BuOH}: \mathrm{H}_{2} \mathrm{O}$ $(1: 1,194 \mathrm{ml})$ were added $\mathrm{K}_{3} \mathrm{Fe}(\mathrm{CN})_{6}(19.2 \mathrm{~g}, 58.2 \mathrm{mmol}), \mathrm{K}_{2} \mathrm{CO}_{3}(8.05 \mathrm{~g}$, $58.2 \mathrm{mmol}), \quad \mathrm{K}_{2} \mathrm{OsO}_{4}(\mathrm{OH})_{4} \quad(71.5 \mathrm{mg}, \quad 0.19 \mathrm{mmol})$ and $(\mathrm{DHQ})_{2} \mathrm{PHAL}$ (151 mg, $0.19 \mathrm{mmol}$ ) at $0{ }^{\circ} \mathrm{C}$ under $\mathrm{N}_{2}$. The reaction mixture was stirred for $4.5 \mathrm{~h}$ at $0{ }^{\circ} \mathrm{C}$. The reaction was quenched with a saturated aqueous $\mathrm{Na}_{2} \mathrm{~S}_{2} \mathrm{O}_{3}$ and the aqueous phase was extracted with $\mathrm{CH}_{2} \mathrm{Cl}_{2}$. The combined organic extracts were dried over anhydrous $\mathrm{Na}_{2} \mathrm{SO}_{4}$ and concentrated in vacuo. The residue was purified by flash column chromatography ( $5: 1$ hexanes/EtOAc) to afford $\mathbf{5}$ (4.66 g, quant.) as a colorless oil. The enantiomeric excess ( $93 \%$ ee) of $\mathbf{5}$ was determined by chiral HPLC analysis (conditions: DAICEL CHIRALPAK AS-3 $(0.46 \mathrm{~cm} \phi \times 25 \mathrm{~cm}$ ), mobile phase of 3:1 hexanes/2-propanol (flow rate of $1.0 \mathrm{ml}$ per min) and detection at $254 \mathrm{~nm}($ rt.)).

$[\alpha]_{\mathrm{D}}^{26}-2.51(c 1.0 \mathrm{MeOH})$; IR $(\mathrm{KBr}) 3478,2976,2842,1706,1606,1512$, $1462 \mathrm{~cm}^{-1} ;{ }^{1} \mathrm{H}-\mathrm{NMR}\left(300 \mathrm{MHz}, \mathrm{CDCl}_{3}\right) \delta 7.98(\mathrm{~d}, J=9.1 \mathrm{~Hz}, 2 \mathrm{H}), 6.90(\mathrm{~d}$, $J=9.1 \mathrm{~Hz}, 2 \mathrm{H}), 4.36(\mathrm{~d}, J=11.3 \mathrm{~Hz}, 1 \mathrm{H}), 4.19(\mathrm{~d}, J=11.3 \mathrm{~Hz}, 1 \mathrm{H}), 3.85(\mathrm{~s}, 3 \mathrm{H})$, $3.57(\mathrm{~d}, J=11.6 \mathrm{~Hz}, 1 \mathrm{H}), 3.46(\mathrm{~d}, J=11.4 \mathrm{~Hz}, 1 \mathrm{H}), 2.95$ (brs, $1 \mathrm{H}), 1.26(\mathrm{~s}, 3 \mathrm{H})$; ${ }^{13} \mathrm{C}-\mathrm{NMR}\left(75.0 \mathrm{MHz}, \mathrm{CDCl}_{3}\right) \delta 166.9,163.7,131.8,121.8,113.8,72.2,68.0$, 66.9, 55.5, 21.3; HRMS (ESI ${ }^{+}$, TFA-Na) calcd for $\mathrm{C}_{12} \mathrm{H}_{16} \mathrm{NaO}_{5} 263.0895$ $[\mathrm{M}+\mathrm{Na}]^{+}$, found $\mathrm{m} / \mathrm{z} 263.0902$.

(R)-2-Methylbutane-1,2-diol (6). To a solution of $5(1.58 \mathrm{~g}, 6.59 \mathrm{mmol})$ in $\mathrm{CH}_{2} \mathrm{Cl}_{2}(66 \mathrm{ml})$ were added $\mathrm{Et}_{3} \mathrm{~N}(1.80 \mathrm{ml}, 13.2 \mathrm{mmol}), \mathrm{Me} 3 \mathrm{~N} \cdot \mathrm{HCl}(63.0 \mathrm{mg}$, $0.66 \mathrm{mmol})$ and $\mathrm{TsCl}(1.90 \mathrm{~g}, 9.89 \mathrm{mmol})$ at $\mathrm{rt}$ under $\mathrm{N}_{2}$. The reaction mixture was stirred for $3 \mathrm{~h}$ at rt. The reaction was quenched with $\mathrm{H}_{2} \mathrm{O}$ and the aqueous phase was extracted with $\mathrm{CH}_{2} \mathrm{Cl}_{2}$. The combined organic extracts were dried over anhydrous $\mathrm{Na}_{2} \mathrm{SO}_{4}$ and concentrated in vacuo. The residue was purified by flash column chromatography (1:1 hexanes/EtOAc) to afford the corresponding tosylate $(2.57 \mathrm{~g}, 99 \%)$ as a colorless oil.

$[\alpha]_{\mathrm{D}}^{26}+1.94\left(c 1.0 \mathrm{CHCl}_{3}\right)$; IR (KBr) 3519, 2982, 2842, 1712, 1604, 1511, $1462 \mathrm{~cm}^{-1} ;{ }^{1} \mathrm{H}-\mathrm{NMR}\left(400 \mathrm{MHz}, \mathrm{CDCl}_{3}\right) \delta 7.87(\mathrm{~d}, J=9.0 \mathrm{~Hz}, 2 \mathrm{H}), 7.76$ $(\mathrm{d}, J=9.0 \mathrm{~Hz}, 2 \mathrm{H}), 7.26(\mathrm{~d}, J=7.8 \mathrm{~Hz}, 2 \mathrm{H}), 6.90(\mathrm{~d}, J=8.8 \mathrm{~Hz}, 2 \mathrm{H}), 4.36$ $(\mathrm{d}, J=11.3 \mathrm{~Hz}, 2 \mathrm{H}), 4.00(\mathrm{~d}, J=8.0 \mathrm{~Hz}, 2 \mathrm{H}), 3.88(\mathrm{~s}, 3 \mathrm{H}), 2.35(\mathrm{~s}, 3 \mathrm{H}), 1.28$ $(\mathrm{s}, 3 \mathrm{H}) ;{ }^{13} \mathrm{C}-\mathrm{NMR}\left(100 \mathrm{MHz}, \mathrm{CDCl}_{3}\right) \delta 166.1,163.7,145.2,132.3,131.8,130.0$, 128.0, 121.7, 113.7, 72.8, 70.8, 67.7, 55.5, 21.6, 21.6; HRMS (ESI ${ }^{+}$, TFA-Na) calcd for $\mathrm{C}_{19} \mathrm{H}_{22} \mathrm{NaO}_{7} \mathrm{~S} 417.0984[\mathrm{M}+\mathrm{Na}]^{+}$, found $\mathrm{m} / z 417.0971$.

To a solution of CuCN $(1.79 \mathrm{~g}, 19.9 \mathrm{mmol})$ in THF $(20 \mathrm{ml})$ was added MeLi $(1.07 \mathrm{M}$ in diethyl ether, $37.3 \mathrm{ml}, 39.9 \mathrm{mmol})$ at $-78^{\circ} \mathrm{C}$ under Ar. After stirring for $20 \mathrm{~min}$ at $0{ }^{\circ} \mathrm{C}$, a solution of the tosylate $(1.57 \mathrm{~g}, 3.99 \mathrm{mmol})$ in THF $(20 \mathrm{ml})$ was added to the reaction mixture at $-78^{\circ} \mathrm{C}$. The reaction was stirred for $30 \mathrm{~min}$ at $-78^{\circ} \mathrm{C}$, then allowed to warm to $0^{\circ} \mathrm{C}$ and stirred for $20 \mathrm{~min}$. The reaction was quenched with a saturated aqueous $\mathrm{NH}_{4} \mathrm{Cl}$ and the aqueous phase was extracted with EtOAc. The combined organic extracts were dried over anhydrous $\mathrm{Na}_{2} \mathrm{SO}_{4}$ and concentrated in vacuo. The resulting residue was dissolved in $\mathrm{MeOH}(40 \mathrm{ml})$ and $\mathrm{K}_{2} \mathrm{CO}_{3}(276 \mathrm{mg}, 1.99 \mathrm{mmol})$ was added. After stirring for $14 \mathrm{~h}$ at $\mathrm{rt}$, the reaction mixture was concentrated in vacuo. The residue was purified by flash column chromatography (5:1 hexanes/EtOAc) to give 6 (373 mg, 90\% over 2 steps) as a colorless oil.

$[\alpha]_{\mathrm{D}}^{25}+4.90\left(\right.$ c $\left.1.0 \mathrm{CHCl}_{3}\right)$; IR ( $\left.\mathrm{KBr}\right) 3424,2926,2856,1654,1462 \mathrm{~cm}^{-1} ;{ }^{1} \mathrm{H}-$ $\operatorname{NMR}\left(300 \mathrm{MHz}, \mathrm{CDCl}_{3}\right) \delta 3.34(\mathrm{~d}, J=11.1 \mathrm{~Hz}, 1 \mathrm{H}), 3.37(\mathrm{~d}, J=11.1 \mathrm{~Hz}, 1 \mathrm{H})$, 3.10 (brs, $1 \mathrm{H}), 2.71$ (brs, $1 \mathrm{H}), 1.50(\mathrm{q}, J=7.3 \mathrm{~Hz}, 2 \mathrm{H}), 1.11(\mathrm{~s}, 3 \mathrm{H}), 0.89$ $(\mathrm{t}, J=7.6 \mathrm{~Hz}, 3 \mathrm{H}) ;{ }^{13} \mathrm{C}-\mathrm{NMR}\left(75.0 \mathrm{MHz}, \mathrm{CDCl}_{3}\right) \delta 73.3,69.3,31.0,22.4,8.03$.

(R)-2-((4-Methoxybenzyl)oxy)-2-methylbutanal (3). To a solution of 6 (776 mg, $7.46 \mathrm{mmol})$ in $\mathrm{CH}_{2} \mathrm{Cl}_{2}(30 \mathrm{ml})$ were added PPTS $(93.7 \mathrm{mg}, 0.37 \mathrm{mmol})$ and $p$ anisaldehyde dimethylacetal $(1.27 \mathrm{ml}, 7.46 \mathrm{mmol})$ at $\mathrm{rt}$ under $\mathrm{N}_{2}$. The reaction mixture was stirred for $6 \mathrm{~h}$ at rt. The reaction was quenched with $\mathrm{H}_{2} \mathrm{O}$ and the aqueous phase was extracted with EtOAc. The combined organic extracts were 
dried over anhydrous $\mathrm{Na}_{2} \mathrm{SO}_{4}$ and concentrated in vacuo. The residue was purified by flash column chromatography (50:1 hexanes/EtOAc) to afford the corresponding $p$-methoxybenzylidene acetal (1.66g, quant.) as a colorless oil.

$[\alpha]_{\mathrm{D}}^{26}+1.40\left(c \quad 1.0 \quad \mathrm{CHCl}_{3}\right)$; IR (KBr) 2969, 2929, 2874, 1614, 1515, $1461 \mathrm{~cm}^{-1} ;{ }^{1} \mathrm{H}-\mathrm{NMR}\left(300 \mathrm{MHz}, \mathrm{CDCl}_{3}\right) \delta 7.42(\mathrm{~d}, J=8.8 \mathrm{~Hz}, 2 \mathrm{H}), 6.90(\mathrm{~d}$, $J=8.8 \mathrm{~Hz}, 2 \mathrm{H}), 5.82(\mathrm{~s}, 1 \mathrm{H}), 3.83(\mathrm{~s}, 2 \mathrm{H}), 3.81(\mathrm{~s}, 3 \mathrm{H}), 1.77-1.68(\mathrm{~m}, 2 \mathrm{H}), 1.38$ (s, 3H), $1.00(\mathrm{t}, J=7.5 \mathrm{~Hz}, 3 \mathrm{H}) ;{ }^{13} \mathrm{C}-\mathrm{NMR}\left(75.0 \mathrm{MHz}, \mathrm{CDCl}_{3}\right) \delta 160.4,130.5$, $128.1,113.7,103.6,81.7,75.4,55.3,31.2,24.3,8.54$; HRMS (ESI ${ }^{+}$, TFA-Na) calcd for $\mathrm{C}_{13} \mathrm{H}_{19} \mathrm{O}_{3:} 223.1334[\mathrm{M}+\mathrm{H}]^{+}$, found $\mathrm{m} / z 223.1342$.

To a solution of the $p$-methoxybenzylidene acetal $(697 \mathrm{mg}, 3.14 \mathrm{mmol})$ in $\mathrm{CH}_{2} \mathrm{Cl}_{2}(31 \mathrm{ml})$ was added DIBAL $(1.02 \mathrm{M}$ in hexanes, $9.00 \mathrm{ml}, 9.41 \mathrm{mmol})$ at $0{ }^{\circ} \mathrm{C}$ under $\mathrm{N}_{2}$. After stirring for $1 \mathrm{~h}$ at $0{ }^{\circ} \mathrm{C}$, the reaction was cautiously quenched with $\mathrm{MeOH}$, diluted with $\mathrm{CH}_{2} \mathrm{Cl}_{2}$ and treated with celite $(2.80 \mathrm{~g})$ and $\mathrm{Na}_{2} \mathrm{SO}_{4} \cdot 10 \mathrm{H}_{2} \mathrm{O}(2.80 \mathrm{~g})$. The mixture was allowed to warm to rt and stirred for $2 \mathrm{~h}$. It was then filtered through a pad of celite and the filtrate was concentrated. The residue was purified by flash column chromatography (10:1 hexanes/ EtOAc) to give the corresponding alcohol $(615 \mathrm{mg}, 87 \%)$ as a colorless oil.

$[\alpha]_{\mathrm{D}}^{26}+1.40(c 1.0 \mathrm{MeOH}) ; \mathrm{IR}(\mathrm{KBr}) 3444,2970,2936,2879,2839,1613$, $1514 \mathrm{~cm}^{-1} ;{ }^{1} \mathrm{H}-\mathrm{NMR}\left(300 \mathrm{MHz}, \mathrm{CDCl}_{3}\right) \delta 7.27(\mathrm{~d}, J=8.7 \mathrm{~Hz}, 2 \mathrm{H}), 6.89$ $(\mathrm{d}, J=8.7 \mathrm{~Hz}, 2 \mathrm{H}), 4.37(\mathrm{~s}, 2 \mathrm{H}), 3.81(\mathrm{~s}, 3 \mathrm{H}), 3.59(\mathrm{~d}, J=4.8 \mathrm{~Hz}, 1 \mathrm{H}), 3.55$ (d, $J=5.0 \mathrm{~Hz}, 1 \mathrm{H}), 2.06$ (brs, $1 \mathrm{H}), 1.66(\mathrm{dq}, J=7.6,2.3 \mathrm{~Hz}, 1 \mathrm{H}), 1.23(\mathrm{~s}, 3 \mathrm{H})$, $0.94(\mathrm{t}, J=7.5 \mathrm{~Hz}, 3 \mathrm{H}) ;{ }^{13} \mathrm{C}-\mathrm{NMR}\left(75.0 \mathrm{MHz}, \mathrm{CDCl}_{3}\right) \delta 159.0,131.2,129.0$, $129.0,113.9,113.9,77.8,66.9,63.3,55.3,27.8,19.7,8.09$; HRMS (FAB, $m$-NBA) calcd for $\mathrm{C}_{13} \mathrm{H}_{19} \mathrm{O}_{3} 223.1334[\mathrm{M}+\mathrm{H}]^{+}$, found $m / z 223.1338$.

To a solution of the alcohol $(239 \mathrm{mg}, 0.94 \mathrm{mmol})$ in $\mathrm{CH}_{2} \mathrm{Cl}_{2}(9.4 \mathrm{ml})$ were added DMSO $(666 \mu \mathrm{l}, 9.37 \mathrm{mmol}), \mathrm{Et}_{3} \mathrm{~N}(1.31 \mathrm{ml}, 9.37 \mathrm{mmol})$ and $\mathrm{SO}_{3} \cdot \mathrm{pyr}$ ( $895 \mathrm{mg}, 5.62 \mathrm{mmol}$ ) at $\mathrm{rt}$ under $\mathrm{N}_{2}$. The reaction mixture was stirred for $3.5 \mathrm{~h}$ at rt. The reaction was quenched with $\mathrm{H}_{2} \mathrm{O}$ and the aqueous phase was extracted with $\mathrm{CH}_{2} \mathrm{Cl}_{2}$. The combined organic extracts were dried over anhydrous $\mathrm{Na}_{2} \mathrm{SO}_{4}$ and concentrated in vacuo. The residue was purified by flash column chromatography (50:1 hexanes/EtOAc) to furnish $3(188 \mathrm{mg}, 89 \%)$ as a colorless oil.

$[\alpha]_{\mathrm{D}}^{22}+6.30$ (c $\left.1.0 \mathrm{CHCl}_{3}\right)$; IR (KBr) 2974, 2937, 2838, 1734, 1613, $1514 \mathrm{~cm}^{-1} ;{ }^{1} \mathrm{H}-\mathrm{NMR}\left(300 \mathrm{MHz}, \mathrm{CDCl}_{3}\right) \delta 9.62(\mathrm{~s}, 1 \mathrm{H}), 7.27(\mathrm{~d}, J=8.8 \mathrm{~Hz}, 2 \mathrm{H})$, $6.87(\mathrm{~d}, J=8.8 \mathrm{~Hz}, 2 \mathrm{H}), 4.39(\mathrm{~d}, J=17.6 \mathrm{~Hz}, 1 \mathrm{H}), 4.35(\mathrm{~d}, J=17.6 \mathrm{~Hz}, 1 \mathrm{H}), 3.78(\mathrm{~s}$, $3 \mathrm{H}), 1.81-1.63(\mathrm{~m}, 2 \mathrm{H}) 1.29(\mathrm{~s}, 3 \mathrm{H}), 0.91(\mathrm{t}, J=7.6 \mathrm{~Hz}, 3 \mathrm{H}) ;{ }^{13} \mathrm{C}-\mathrm{NMR}(75.0 \mathrm{MHz}$, $\left.\mathrm{CDCl}_{3}\right) \delta 205.3,159.3,130.4,129.1,113.9,82.8,65.9,55.3,27.7,17.7,7.30$; HRMS (FAB, $m$-NBA) calcd for $\mathrm{C}_{13} \mathrm{H}_{18} \mathrm{NaO}_{3} 245.1154[\mathrm{M}+\mathrm{Na}]^{+}$, found $m / z 245.1162$.

(E)-7-((tert-Butyldimethylsilyl)oxy)hept-2-en-1-ol (7). To a solution of $\mathrm{NaH}$ $(1.94 \mathrm{~g}, 47.5 \mathrm{mmol})$ in THF $(158 \mathrm{ml})$ were added 1,5 -pentanediol $(5.00 \mathrm{ml}$, $47.5 \mathrm{mmol})$ and TBSCl $(7.31 \mathrm{~g}, 47.5 \mathrm{mmol})$ at $0{ }^{\circ} \mathrm{C}$ under $\mathrm{N}_{2}$. The reaction mixture was stirred for $5 \mathrm{~min}$ at $0{ }^{\circ} \mathrm{C}$, then allowed to warm to rt and stirred for $2.5 \mathrm{~h}$. The reaction was quenched with $\mathrm{H}_{2} \mathrm{O}$ at $0{ }^{\circ} \mathrm{C}$ and the aqueous phase was extracted with $\mathrm{CH}_{2} \mathrm{Cl}_{2}$. The combined organic extracts were dried over anhydrous $\mathrm{Na}_{2} \mathrm{SO}_{4}$ and concentrated in vacuo. The residue was purified by flash column chromatography (5:1 hexanes/EtOAc) to afford the corresponding TBS ether $(8.30 \mathrm{~g}, 80 \%)$ as a colorless oil.

IR (KBr) 3359, 2933, 2860, 1468, 1254, $1100 \mathrm{~cm}^{-1} ;{ }^{1} \mathrm{H}-\mathrm{NMR}(400 \mathrm{MHz}$, $\left.\mathrm{CDCl}_{3}\right) \delta$ 3.64-3.59 (m, 4H), 1.58-1.52 (m, 4H), 1.40-1.39 (m, $\left.2 \mathrm{H}\right), 0.88$ (s, 9H), $0.04(\mathrm{~s}, 6 \mathrm{H}) ;{ }^{13} \mathrm{C}-\mathrm{NMR}\left(100 \mathrm{MHz}, \mathrm{CDCl}_{3}\right) \delta 63.2,62.9,32.5,32.5,26.0$, $22.0,18.4,-2.28$; HRMS (FAB, $m$-NBA) calcd for $\mathrm{C}_{11} \mathrm{H}_{27} \mathrm{O}_{2} \mathrm{Si} 219.1780$ $[\mathrm{M}+\mathrm{H}]^{+}$, found $m / z 219.1776$.

To a solution of the TBS ether $(4.00 \mathrm{~g}, 18.3 \mathrm{mmol})$ in $\mathrm{CH}_{2} \mathrm{Cl}_{2}(183 \mathrm{ml})$ were added iodobenzene diacetate $(8.86 \mathrm{~g}, 27.5 \mathrm{mmol})$ and TEMPO $(573 \mathrm{mg}$, $3.67 \mathrm{mmol}$ ) at $\mathrm{rt}$ under $\mathrm{N}_{2}$. The reaction mixture was stirred for $5 \mathrm{~h}$ at $\mathrm{rt}$. The reaction was quenched with an aqueous $\mathrm{Na}_{2} \mathrm{~S}_{2} \mathrm{O}_{3}$ solution, and the aqueous phase was extracted with $\mathrm{CH}_{2} \mathrm{Cl}_{2}$. The combined organic extracts were dried over anhydrous $\mathrm{Na}_{2} \mathrm{SO}_{4}$ and concentrated in vacuo. The residue was purified by flash column chromatography (50:1 hexanes/EtOAc) to afford the corresponding aldehyde (3.96 g, quant.) as a colorless oil.

IR (KBr) 2952, 2933, 2859, 1727, 1468, 1254, $1102 \mathrm{~cm}^{-1} ; \quad{ }^{1} \mathrm{H}-\mathrm{NMR}$ $\left(400 \mathrm{MHz}, \mathrm{CDCl}_{3}\right) \delta 9.75(\mathrm{~s}, 1 \mathrm{H}), 3.61(\mathrm{t}, J=6.2 \mathrm{~Hz}, 2 \mathrm{H}), 2.46-2.42(\mathrm{~m}, 2 \mathrm{H})$, $1.70-1.65(\mathrm{~m}, 2 \mathrm{H}), 1.57-1.51(\mathrm{~m}, 2 \mathrm{H}), 0.87(\mathrm{~s}, 9 \mathrm{H}), 0.03(\mathrm{~s}, 6 \mathrm{H}) ;{ }^{13} \mathrm{C}-\mathrm{NMR}$ $\left(100 \mathrm{MHz}, \mathrm{CDCl}_{3}\right) \delta 203.7,62.6,43.6,32.1,25.9,18.6,18.3,-5.34$; HRMS (FAB, $m$-NBA) calcd for $\mathrm{C}_{11} \mathrm{H}_{25} \mathrm{O}_{2} \mathrm{Si} 217.1624[\mathrm{M}+\mathrm{H}]^{+}$, found $m / z$ 217.1618.

To a solution of the aldehyde $(3.19 \mathrm{~g}, 14.8 \mathrm{mmol})$ in benzene $(148 \mathrm{ml})$ was added ethyl(triphenylphosphoranylidene)acetate $(7.71 \mathrm{~g}, 22.1 \mathrm{mmol})$ under $\mathrm{N}_{2}$.
The reaction mixture was stirred for $3 \mathrm{~h}$ at $80^{\circ} \mathrm{C}$. The reaction was concentrated in vacuo. The residue was purified by flash column chromatography (80:1 hexanes/EtOAc) to give the corresponding $\alpha, \beta$-unsaturated ester $(4.19 \mathrm{~g}$, quant.) as a colorless oil.

IR (KBr) 2933，2859，1724，1655，1468，1259，1101 cm ${ }^{-1} ;{ }^{1} \mathrm{H}-\mathrm{NMR}$ $\left(400 \mathrm{MHz}, \mathrm{CDCl}_{3}\right) \delta 6.94(\mathrm{dt}, J=15.7,7.0 \mathrm{~Hz}, 1 \mathrm{H}), 5.79(\mathrm{dt}, J=15.7,1.6 \mathrm{~Hz}$, $1 \mathrm{H}), 4.16(\mathrm{q}, J=7.2 \mathrm{~Hz}, 2 \mathrm{H}), 3.59(\mathrm{t}, J=6.1 \mathrm{~Hz}, 2 \mathrm{H}), 2.21-2.19(\mathrm{~m}, 2 \mathrm{H})$, $1.52-1.49(\mathrm{~m}, 4 \mathrm{H}), 1.26(\mathrm{t}, J=7.1 \mathrm{~Hz}, 3 \mathrm{H}), 0.87(\mathrm{~s}, 9 \mathrm{H}), 0.02(\mathrm{~s}, 6 \mathrm{H})$; ${ }^{13} \mathrm{C}-\mathrm{NMR}\left(100 \mathrm{MHz}, \mathrm{CDCl}_{3}\right) \delta 166.7,149.1,121.4,62.7,60.1,32.2,31.9$, 25.9, 24.4, 18.3, 14.3, -5.32; HRMS (ESI ${ }^{+}$, TFA-Na) calcd for $\mathrm{C}_{15} \mathrm{H}_{30} \mathrm{NaO}_{3} \mathrm{Si}$ $309.1862[\mathrm{M}+\mathrm{Na}]^{+}$, found $\mathrm{m} / \mathrm{z} 309.1877$.

To a solution of the $\alpha, \beta$-unsaturated ester $(1.82 \mathrm{~g}, 8.02 \mathrm{mmol})$ in $\mathrm{CH}_{2} \mathrm{Cl}_{2}$ $(80 \mathrm{ml})$ was added DIBAL $(1.02 \mathrm{M}$ in hexanes, $15.7 \mathrm{ml}, 16.0 \mathrm{mmol})$ at $0{ }^{\circ} \mathrm{C}$ under $\mathrm{N}_{2}$. After stirring for $1 \mathrm{~h}$, the reaction was cautiously quenched with $\mathrm{MeOH}$, diluted with $\mathrm{CH}_{2} \mathrm{Cl}_{2}$ and treated with celite $(5.90 \mathrm{~g})$ and $\mathrm{Na}_{2} \mathrm{SO}_{4} \cdot 10 \mathrm{H}_{2} \mathrm{O}(5.90 \mathrm{~g})$. The mixture was allowed to warm to $\mathrm{rt}$ and stirred for $2 \mathrm{~h}$, then filtered through a pad of celite and the filtrate was concentrated. The residue was purified by flash column chromatography (20:1 hexanes/ EtOAc) to furnish 7 (1.96g, quant.) as a colorless oil.

IR (KBr) 3339, 2932, 2859, 1467, 1254, $1101 \mathrm{~cm}^{-1}$; ${ }^{1} \mathrm{H}-\mathrm{NMR}(300 \mathrm{MHz}$, $\left.\mathrm{CDCl}_{3}\right) \delta 5.68-5.63(\mathrm{~m}, 2 \mathrm{H}), 4.07(\mathrm{~d}, J=4.7 \mathrm{~Hz}, 2 \mathrm{H}), 3.60(\mathrm{t}, J=6.3 \mathrm{~Hz}, 2 \mathrm{H}), 2.09$ $2.02(\mathrm{~m}, 2 \mathrm{H}), 1.55-1.39(\mathrm{~m}, 4 \mathrm{H}), 0.88(\mathrm{~s}, 9 \mathrm{H}), 0.04(\mathrm{~s}, 6 \mathrm{H}) ;{ }^{13} \mathrm{C}-\mathrm{NMR}(75.0 \mathrm{MHz}$, $\left.\mathrm{CDCl}_{3}\right) \delta 133.2,129.1,63.8,63.0,32.3,31.9,26.0,26.0,25.4,18.4,-5.29$; HRMS (FAB, $m$-NBA) calcd for $\mathrm{C}_{13} \mathrm{H}_{29} \mathrm{O}_{2} \mathrm{Si} 245.1937[\mathrm{M}+\mathrm{H}]^{+}$, found $\mathrm{m} / z 245.1934$.

(E)-7-((Tetrahydro-2H-pyran-2-yl)oxy)hept-5-en-1-ol (8). To a solution of 7 (1.96 g, $8.02 \mathrm{mmol})$ in $\mathrm{CH}_{2} \mathrm{Cl}_{2}(40 \mathrm{ml})$ were added PPTS $(202 \mathrm{mg}, 0.80 \mathrm{mmol})$ and DHP $(7.25 \mathrm{ml}, 80.2 \mathrm{mmol})$ at $0^{\circ} \mathrm{C}$ under $\mathrm{N}_{2}$. The reaction mixture was stirred for $6 \mathrm{~h}$ at $0{ }^{\circ} \mathrm{C}$. The reaction was quenched with $\mathrm{H}_{2} \mathrm{O}$ and the aqueous phase was extracted with EtOAc. The combined organic extracts were dried over anhydrous $\mathrm{Na}_{2} \mathrm{SO}_{4}$ and concentrated in vacuo. The residue was purified by flash column chromatography (50:1 hexanes/EtOAc) to afford the corresponding THP ether $(2.63 \mathrm{~g}$, quant.) as a colorless oil.

IR (KBr) 2935, 2859, 1467, 1254, $1102 \mathrm{~cm}^{-1} ;{ }^{1} \mathrm{H}-\mathrm{NMR}\left(400 \mathrm{MHz}, \mathrm{CDCl}_{3}\right) \delta$ 5.72-5.67 (m, $1 \mathrm{H}), 5.60-5.54(\mathrm{~m}, 1 \mathrm{H}), 4.64-4.62(\mathrm{~m}, 1 \mathrm{H}), 4.17$ (ddd, $J=11.9$, $5.7,1.2 \mathrm{~Hz}, 1 \mathrm{H}), 3.92(\mathrm{ddd}, J=11.9,5.7,1.2 \mathrm{~Hz}, 1 \mathrm{H}), 3.90-3.84(\mathrm{~m}, 1 \mathrm{H}), 3.59$ $(\mathrm{t}, J=6.5 \mathrm{~Hz}, 2 \mathrm{H}), 3.51-3.48(\mathrm{~m}, 1 \mathrm{H}), 2.08-2.03(\mathrm{~m}, 2 \mathrm{H}), 1.82-1.56(\mathrm{~m}, 6 \mathrm{H})$ $1.55-1.39(\mathrm{~m}, 4 \mathrm{H}), 0.88(\mathrm{~s}, 9 \mathrm{H}), 0.03(\mathrm{~s}, 6 \mathrm{H}) ;{ }^{13} \mathrm{C}-\mathrm{NMR}\left(100 \mathrm{MHz}, \mathrm{CDCl}_{3}\right)$ $\delta$ 134.5, 126.3, 97.8, 67.9, 63.1, 62.3, 32.4, 32.1, 30.7, 26.0, 25.5, 25.3, 19.6, 18.4, -5.26; HRMS (ESI ${ }^{+}$, TFA-Na) calcd for $\mathrm{C}_{18} \mathrm{H}_{36} \mathrm{NaO}_{3} \mathrm{Si} 351.2325[\mathrm{M}+\mathrm{Na}]^{+}$, found $\mathrm{m} / \mathrm{z} 351.2331$.

To a solution of the THP ether $(2.11 \mathrm{~g}, 6.42 \mathrm{mmol})$ in THF $(64 \mathrm{ml})$ was added TBAF $(7.71 \mathrm{ml}, 7.71 \mathrm{mmol})$ at $\mathrm{rt}$ under $\mathrm{N}_{2}$. The reaction mixture was stirred for $4.5 \mathrm{~h}$ at $\mathrm{rt}$. The reaction was quenched with $\mathrm{H}_{2} \mathrm{O}$, and the aqueous phase was extracted with $\mathrm{CH}_{2} \mathrm{Cl}_{2}$. The combined organic extracts were dried over anhydrous $\mathrm{Na}_{2} \mathrm{SO}_{4}$ and concentrated in vacuo. The residue was purified by flash column chromatography (1:1 hexanes/EtOAc) to give 8 (1.34 g, quant.) as a colorless oil. IR (KBr) 3393, 2939, 2866, 1499, 1351, $1120 \mathrm{~cm}^{-1}$; ${ }^{1} \mathrm{H}-\mathrm{NMR}(300 \mathrm{MHz}$, $\left.\mathrm{CDCl}_{3}\right) \delta 5.72-5.65(\mathrm{~m}, 1 \mathrm{H}), 5.61-5.54(\mathrm{~m}, 1 \mathrm{H}), 4.63-4.60(\mathrm{~m}, 1 \mathrm{H}), 4.16(\mathrm{ddd}$, $J=11.9,5.5,1.1 \mathrm{~Hz}, 1 \mathrm{H}), 3.90$ (ddd, $J=11.9,5.5,1.1 \mathrm{~Hz}, 1 \mathrm{H}), 3.89-3.82$ $(\mathrm{m}, 1 \mathrm{H}), 3.61(\mathrm{t}, J=6.5 \mathrm{~Hz}, 2 \mathrm{H}), 3.50-3.46(\mathrm{~m}, 1 \mathrm{H}), 2.10-2.03(\mathrm{~m}, 2 \mathrm{H})$, $1.83-1.41(\mathrm{~m}, 10 \mathrm{H}) ;{ }^{13} \mathrm{C}-\mathrm{NMR}\left(75.0 \mathrm{MHz}, \mathrm{CDCl}_{3}\right) \delta 133.0,127.0,97.8,67.8$, $62.7,62.2,32.2,32.0,30.6,25.4,25.2,19.5$; HRMS (FAB, $m$-NBA) calcd for $\mathrm{C}_{12} \mathrm{H}_{22} \mathrm{NaO}_{3} 273.1467[\mathrm{M}+\mathrm{Na}]^{+}$, found $m / z 273.1467$.

(E)-2-((7-Iodohept-2-en-1-yl)oxy)tetrahydro-2H-pyran (4). To a solution of 8 $(657 \mathrm{mg}, 3.07 \mathrm{mmol})$ in $\mathrm{CH}_{2} \mathrm{Cl}_{2}(31 \mathrm{ml})$ were added $\mathrm{Et}_{3} \mathrm{~N}(855 \mu \mathrm{l}, 6.14 \mathrm{mmol})$, $\mathrm{Me}_{3} \mathrm{~N} \cdot \mathrm{HCl}(29.3 \mathrm{mg}, 0.31 \mathrm{mmol})$ and $\mathrm{MsCl}(356 \mu \mathrm{l}, 4.61 \mathrm{mmol})$ at $0{ }^{\circ} \mathrm{C}$ under $\mathrm{N}_{2}$. The reaction mixture was allowed to warm to rt and stirred for $3 \mathrm{~h}$. The reaction was quenched with $\mathrm{H}_{2} \mathrm{O}$ and the aqueous phase was extracted with $\mathrm{CH}_{2} \mathrm{Cl}_{2}$. The combined organic extracts were dried over anhydrous $\mathrm{Na}_{2} \mathrm{SO}_{4}$ and concentrated in vacuo. The residue was purified by flash column chromatography (10:1 hexanes/EtOAc) to afford the corresponding mesylate $(837 \mathrm{mg}$, $93 \%)$ as a colorless oil.

IR (KBr) 2940, 2866, 1354, 1174, $1121 \mathrm{~cm}^{-1},{ }^{1} \mathrm{H}-\mathrm{NMR}\left(400 \mathrm{MHz}, \mathrm{CDCl}_{3}\right)$

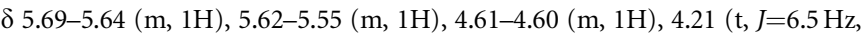
$2 \mathrm{H}), 4.17$ (ddd, $J=12.0,5.3,1.2 \mathrm{~Hz}, 1 \mathrm{H}), 3.91$ (ddd, $J=12.0,5.3,1.2 \mathrm{~Hz}, 1 \mathrm{H}$ ), 
3.88-3.47 (m, 1H), 3.51-3.47 (m, 1H), $2.99(\mathrm{~s}, 3 \mathrm{H}), 2.12-2.06(\mathrm{~m}, 2 \mathrm{H}), 1.84-$ $1.46(\mathrm{~m}, 10 \mathrm{H}) ;{ }^{13} \mathrm{C}-\mathrm{NMR}\left(100 \mathrm{MHz}, \mathrm{CDCl}_{3}\right) \delta 133.0,127.0,97.8,69.8,67.6$, $62.2,37.2,31.5,30.5,28.5,25.3,24.7,19.4$; HRMS (ESI ${ }^{+}$, TFA-Na) calcd for $\mathrm{C}_{13} \mathrm{H}_{24} \mathrm{NaO}_{5} \mathrm{~S} 315.1242[\mathrm{M}+\mathrm{Na}]^{+}$, found $m / z$ 315.1206.

To a solution of $\mathrm{NaI}(2.42 \mathrm{~g}, 16.2 \mathrm{mmol})$ in acetone $(216 \mathrm{ml})$ was added the mesylate $(3.15 \mathrm{~g}, 10.8 \mathrm{mmol})$ under $\mathrm{N}_{2}$. The reaction mixture was stirred for $8.5 \mathrm{~h}$ at reflux. After cooling to rt, the reaction was quenched with $\mathrm{H}_{2} \mathrm{O}$ and the aqueous phase was extracted with EtOAc. The combined organic extracts were dried over anhydrous $\mathrm{Na}_{2} \mathrm{SO}_{4}$ and concentrated in vacuo. The residue was purified by flash column chromatography (80:1 hexanes/EtOAc) to give 4 ( $3.49 \mathrm{~g}$, quant.) as a colorless oil.

IR (KBr) 2941, 2869, 1451, 1350, $1121 \mathrm{~cm}^{-1}$; ${ }^{1} \mathrm{H}-\mathrm{NMR}\left(300 \mathrm{MHz}, \mathrm{CDCl}_{3}\right)$ $\delta 5.71-5.54(\mathrm{~m}, 2 \mathrm{H}), 4.61(\mathrm{dd}, J=4.3,2.9 \mathrm{~Hz}, 1 \mathrm{H}), 4.17(\mathrm{ddd}, J=12.0,5.4$, $1.0 \mathrm{~Hz}, 1 \mathrm{H}), 3.91$ (ddd, $J=12.0,5.3,1.2 \mathrm{~Hz}, 1 \mathrm{H}), 3.88-3.82(\mathrm{~m}, 1 \mathrm{H}), 3.53-3.47$ $(\mathrm{m}, 1 \mathrm{H}), 3.17(\mathrm{t}, J=7.0 \mathrm{~Hz}, 2 \mathrm{H}), 2.10-2.03(\mathrm{~m}, 2 \mathrm{H}), 1.87-1.44(\mathrm{~m}, 10 \mathrm{H})$; ${ }^{13} \mathrm{C}$-NMR $\left(75.0 \mathrm{MHz}, \mathrm{CDCl}_{3}\right) \delta 133.5,126.8,97.8,67.7,62.3,33.0,31.2,30.6$, 29.8, 25.5, 19.6, 6.80; HRMS (FAB, $m$-NBA): calcd for $\mathrm{C}_{12} \mathrm{H}_{21} \mathrm{NaO}_{2} \mathrm{I} 347.0484$ $[\mathrm{M}+\mathrm{Na}]^{+}$, found $\mathrm{m} / \mathrm{z} 347.0484$.

(3R,E)-3-((4-Methoxybenzyl)oxy)-3-methyl-11-((tetrahydro-2H-pyran-2-yl)oxy)un dec-9-en-4-ol (9). To a solution of iodide $4(844 \mathrm{mg}, 3.8 \mathrm{mmol})$ in pentane $(22 \mathrm{ml})$ were added $\mathrm{Et}_{2} \mathrm{O}(8.2 \mathrm{ml})$ and $t$ - $\mathrm{BuLi}(1.55 \mathrm{M}$ in pentane, $5.64 \mathrm{ml}$, $8.74 \mathrm{mmol})$ at $-78^{\circ} \mathrm{C}$ under Ar. After stirring for $15 \mathrm{~min}$ at $-78^{\circ} \mathrm{C}$, a solution of aldehyde $3(1.35 \mathrm{~g}, 4.18 \mathrm{mmol})$ in $\mathrm{Et}_{2} \mathrm{O}(7.0 \mathrm{ml})$ was added. The reaction mixture was allowed to warm to $0{ }^{\circ} \mathrm{C}$ and stirred for $3 \mathrm{~h}$. The mixture was quenched with an aqueous $\mathrm{NH}_{4} \mathrm{Cl}$ solution and the aqueous phase was extracted with EtOAc. The combined organic extracts were dried over anhydrous $\mathrm{Na}_{2} \mathrm{SO}_{4}$ and concentrated in vacuo. The residue was purified by flash column chromatography (15:1 hexanes/EtOAc) to afford $\mathbf{9}(1.54 \mathrm{~g}, 96 \%)$ as a colorless oil.

$[\alpha]_{\mathrm{D}}^{22}+3.88\left(c 1.0 \mathrm{CHCl}_{3}\right)$; IR ( $\left.\mathrm{KBr}\right) 3561,2837,1612,1512,1458 \mathrm{~cm}^{-1}$; ${ }^{1} \mathrm{H}-\mathrm{NMR}\left(300 \mathrm{MHz}, \mathrm{CDCl}_{3}\right) \delta 7.26(\mathrm{~d}, J=8.8 \mathrm{~Hz}, 2 \mathrm{H}), 6.88(\mathrm{~d}, J=8.8 \mathrm{~Hz}, 2 \mathrm{H})$, $5.75-5.68(\mathrm{~m}, 1 \mathrm{H}), 5.62-5.55(\mathrm{~m}, 1 \mathrm{H}), 4.64(\mathrm{dd}, J=3.5,3.0 \mathrm{~Hz}, 1 \mathrm{H}), 4.34(\mathrm{~s}, 2 \mathrm{H})$, 4.19 (ddd, $J=11.9,5.6,1.1 \mathrm{~Hz}, 1 \mathrm{H}$ ), 3.92 (ddd, $J=11.9,6.7,0.8 \mathrm{~Hz}, 1 \mathrm{H}$ ), 3.88-3.82 (m, $1 \mathrm{H}), 3.80(\mathrm{~s}, 3 \mathrm{H}), 3.68-3.64(\mathrm{~m}, 1 \mathrm{H}), 3.54-3.47(\mathrm{~m}, 1 \mathrm{H}), 2.17-2.05(\mathrm{~m}, 2 \mathrm{H})$, $1.86-1.15(\mathrm{~m}, 14 \mathrm{H}), 1.14(\mathrm{~s}, 3 \mathrm{H}), 0.92(\mathrm{t}, J=7.5 \mathrm{~Hz}, 3 \mathrm{H}) ;{ }^{13} \mathrm{C}-\mathrm{NMR}(75.0 \mathrm{MHz}$, $\left.\mathrm{CDCl}_{3}\right) \delta 159.0,134.9,131.0,128.9,126.1,113.8,97.7,80.1,73.9,67.8,62.8,62.2$, 55.2, 32.3, 30.8, 30.6, 29.6, 29.1, 26.5, 26.4, 25.4, 19.5, 17.6, 14.1, 7.34; HRMS (ESI ${ }^{+}$, TFA-Na) calcd for $\mathrm{C}_{25} \mathrm{H}_{40} \mathrm{NaO}_{5} 443.2773[\mathrm{M}+\mathrm{Na}]^{+}$, found $\mathrm{m} / z 443.2753$.

\section{(R,E)-11-Hydroxy-3-((4-methoxybenzyl)oxy)-3-methylundec-9-en-4-one}

(10). To a solution of $9(674 \mathrm{mg}, 1.60 \mathrm{mmol})$ in $\mathrm{CH}_{2} \mathrm{Cl}_{2}(16 \mathrm{ml})$ were added NMO (380 mg, $3.21 \mathrm{mmol}$ ), TPAP $(28.2 \mathrm{mg}, 0.08 \mathrm{mmol})$ and $4 \AA$ molecular sieves $(674 \mathrm{mg})$ at $\mathrm{rt}$ under $\mathrm{N}_{2}$. The reaction mixture was stirred for $1.5 \mathrm{~h}$ at $\mathrm{rt}$. The mixture was filtered through a pad of celite. The filtrate was diluted with $\mathrm{H}_{2} \mathrm{O}$ and extracted with $\mathrm{CH}_{2} \mathrm{Cl}_{2}$ followed by EtOAc. The combined organic extracts were dried over anhydrous $\mathrm{Na}_{2} \mathrm{SO}_{4}$ and concentrated in vacuo. The residue was purified by flash column chromatography (40:1 hexanes/ EtOAc) to afford the corresponding ketone $(652 \mathrm{mg}, 97 \%)$ as a colorless oil.

$[\alpha]_{\mathrm{D}}^{27}+7.80\left(c 1.0 \mathrm{CHCl}_{3}\right)$; IR (KBr) 2940, 2868, 1712, 1613, 1514, $1459 \mathrm{~cm}^{-1}$; ${ }^{1} \mathrm{H}-\mathrm{NMR}\left(300 \mathrm{MHz}, \mathrm{CDCl}_{3}\right) \delta 7.27(\mathrm{~d}, J=8.8 \mathrm{~Hz}, 2 \mathrm{H}), 6.89(\mathrm{~d}, J=8.8 \mathrm{~Hz}, 2 \mathrm{H})$, $5.71-5.51(\mathrm{~m}, 1 \mathrm{H}), 5.60-5.51(\mathrm{~m}, 1 \mathrm{H}), 4.63-4.25(\mathrm{~m}, 1 \mathrm{H}), 4.32(\mathrm{~d}, J=10.7 \mathrm{~Hz}, 1 \mathrm{H})$, $4.28(\mathrm{~d}, J=10.7 \mathrm{~Hz}, 1 \mathrm{H}), 4.17$ (ddd, $J=12.0,5.5,1.1 \mathrm{~Hz}, 1 \mathrm{H}$ ), 3.91 (ddd, $J=12.0$, $5.5,1.1 \mathrm{~Hz}, 1 \mathrm{H}), 3.88-3.83(\mathrm{~m}, 1 \mathrm{H}) 3.80(\mathrm{~s}, 3 \mathrm{H}), 3.80-3.46(\mathrm{~m}, 1 \mathrm{H}), 2.64(\mathrm{dt}$, $J=7.3,3.5 \mathrm{~Hz}, 2 \mathrm{H}), 2.09-2.01(\mathrm{~m}, 2 \mathrm{H}), 1.87-1.35(\mathrm{~m}, 10 \mathrm{H}), 1.42-1.35(\mathrm{~m}, 2 \mathrm{H})$, $1.32(\mathrm{~s}, 3 \mathrm{H}), 0.84(\mathrm{t}, J=7.5 \mathrm{~Hz}, 3 \mathrm{H}) ;{ }^{13} \mathrm{C}-\mathrm{NMR}\left(75.0 \mathrm{MHz}, \mathrm{CDCl}_{3}\right) \delta 214.9,159.0$, 134.2, 130.7, 128.6, 126.4, 113.8, 97.8, 84.9, 67.8, 65.1, 62.6, 55.3, 36.8, 32.2, 30.7, 29.3, 28.8, 25.5, 23.0, 19.9, 19.6, 7.89; HRMS (ESI ${ }^{+}$, TFA-Na) calcd for $\mathrm{C}_{25} \mathrm{H}_{38} \mathrm{NaO}_{5} 441.2617[\mathrm{M}+\mathrm{Na}]^{+}$, found $m / z$ 441.2605.

To a solution of the ketone $(597 \mathrm{mg}, 1.43 \mathrm{mmol})$ in $\mathrm{MeOH}(14 \mathrm{ml})$ was added PPTS $\left(359 \mathrm{mg}, 1.43 \mathrm{mmol}\right.$ ) at $\mathrm{rt}$ under $\mathrm{N}_{2}$. The reaction mixture was stirred for $5 \mathrm{~h}$ at rt. The reaction was quenched with $\mathrm{H}_{2} \mathrm{O}$ and the aqueous phase was extracted with $\mathrm{CH}_{2} \mathrm{Cl}_{2}$. The combined organic extracts were dried over anhydrous $\mathrm{Na}_{2} \mathrm{SO}_{4}$ and concentrated in vacuo. The residue was purified by flash column chromatography (10:1 hexanes/EtOAc) to afford $10(475 \mathrm{mg}$, $99 \%)$ as a colorless oil.

$[\alpha]_{\mathrm{D}}^{22}+4.35$ (c $\left.1.0 \mathrm{CHCl}_{3}\right)$; IR (KBr) 3561, 3298, 2866, 1712, 1612, 1513 , $1458 \mathrm{~cm}^{-1}$; ${ }^{1} \mathrm{H}-\mathrm{NMR}\left(300 \mathrm{MHz}, \mathrm{CDCl}_{3}\right) \delta 7.27$ (d, J=8.8 Hz, $\left.2 \mathrm{H}\right), 6.89$ (d, $J=8.8 \mathrm{~Hz}, 2 \mathrm{H}), 5.69-5.58(\mathrm{~m}, 2 \mathrm{H}), 4.32(\mathrm{~d}, J=10.7 \mathrm{~Hz}, 1 \mathrm{H}), 4.29(\mathrm{~d}$, $J=10.7 \mathrm{~Hz}, 1 \mathrm{H}), 4.07-4.05(\mathrm{~m}, 2 \mathrm{H}), 3.80(\mathrm{~s}, 3 \mathrm{H}), 2.64(\mathrm{dt}, J=7.3,3.8 \mathrm{~Hz}$, $2 \mathrm{H}), 2.08-2.01(\mathrm{~m}, 2 \mathrm{H}), 1.87-1.68(\mathrm{~m}, 2 \mathrm{H}), 1.62-1.52(\mathrm{~m}, 2 \mathrm{H}), 1.41-1.34$ (m, $2 \mathrm{H}), 1.32(\mathrm{~s}, 3 \mathrm{H}), 0.84(\mathrm{t}, J=7.5 \mathrm{~Hz}, 3 \mathrm{H}) ;{ }^{13} \mathrm{C}-\mathrm{NMR}\left(75.0 \mathrm{MHz}, \mathrm{CDCl}_{3}\right)$ $\delta$ 215.0, 159.0, 132.8, 130.7, 129.3, 128.6, 113.8, 113.8, 65.1, 63.7, 55.3, 36.8, 32.1, 29.2, 28.8, 23.0, 19.9, 7.90; HRMS (ESI ${ }^{+}$, TFA-Na) calcd for $\mathrm{C}_{20} \mathrm{H}_{30} \mathrm{NaO}_{4}$ $357.2042[\mathrm{M}+\mathrm{Na}]^{+}$, found $\mathrm{m} / \mathrm{z}$ 357.2031.

(R)-8-((2S,3S)-3-(Hydroxymethyl)oxiran-2-yl)-3-((4-methoxybenzyl)oxy)-3-methyloctan-4-one (2). To a suspension of $4 \AA$ molecular sieves (134 mg) and (+)-DET $(177 \mu \mathrm{l}, 1.03 \mathrm{mmol})$ in $\mathrm{CH}_{2} \mathrm{Cl}_{2}(5.0 \mathrm{ml})$ was added $\mathrm{Ti}(\mathrm{O} i \mathrm{Pr})_{4}(305 \mu \mathrm{l}, 1.03 \mathrm{mmol})$ at $-20^{\circ} \mathrm{C}$ under Ar. After stirring for $0.5 \mathrm{~h}, t-\mathrm{BuOOH}(413 \mu \mathrm{l}, 2.06 \mathrm{mmol})$ was slowly added to the suspension at $-20^{\circ} \mathrm{C}$ and the resulting mixture was stirred for $0.5 \mathrm{~h}$. A solution of $\mathbf{1 0}(335 \mathrm{mg}, 1.03 \mathrm{mmol})$ in $\mathrm{CH}_{2} \mathrm{Cl}_{2}(5.3 \mathrm{ml})$ was then added dropwise to the reaction mixture and the mixture was stirred for $18 \mathrm{~h}$ at $-20^{\circ} \mathrm{C}$. The reaction was quenched with $\mathrm{Me}_{2} \mathrm{~S}(105 \mu \mathrm{l}, 1.43 \mathrm{mmol})$, diluted with $\mathrm{CH}_{2} \mathrm{Cl}_{2}$ and treated with celite $(1.10 \mathrm{~g})$ and $\mathrm{Na}_{2} \mathrm{SO}_{4} \cdot 10 \mathrm{H}_{2} \mathrm{O}(1.10 \mathrm{~g})$. The suspension was allowed to warm to $\mathrm{rt}$ and then stirred for $2 \mathrm{~h}$. The resulting mixture was filtered through a pad of celite and the filtrate was concentrated in vacuo. The residue was purified by flash column chromatography (5:1 hexanes/ EtOAc) to afford $2(311 \mathrm{mg}, 86 \%)$ as a colorless oil.

$[\alpha]_{\mathrm{D}}^{28}-2.89\left(c 1.0 \mathrm{CHCl}_{3}\right)$; IR (KBr) 3460, 2935, 1711, 1613, 1514, $1461 \mathrm{~cm}^{-1}$; ${ }^{1} \mathrm{H}-\mathrm{NMR}\left(400 \mathrm{MHz}, \mathrm{CDCl}_{3}\right) \delta 7.27(\mathrm{~d}, J=8.8 \mathrm{~Hz}, 2 \mathrm{H}), 6.89(\mathrm{~d}, J=8.8 \mathrm{~Hz}, 2 \mathrm{H})$, $4.33(\mathrm{~d}, J=10.7 \mathrm{~Hz}, 1 \mathrm{H}), 4.29(\mathrm{~d}, J=10.7 \mathrm{~Hz}, 1 \mathrm{H}), 3.90-3.82(\mathrm{~m}, 1 \mathrm{H}), 3.80(\mathrm{~s}, 3 \mathrm{H})$, $3.64-3.62(\mathrm{~m}, 1 \mathrm{H}), 2.96-2.88(\mathrm{~m}, 2 \mathrm{H}), 2.66(\mathrm{dt}, J=7.2,2.6 \mathrm{~Hz}, 2 \mathrm{H}), 1.88-1.75(\mathrm{~m}$, $2 \mathrm{H}), 1.73-1.54(\mathrm{~m}, 4 \mathrm{H}), 1.48-1.39(\mathrm{~m}, 2 \mathrm{H}), 1.33(\mathrm{~s}, 3 \mathrm{H}), 0.84(\mathrm{t}, J=7.5 \mathrm{~Hz}, 3 \mathrm{H})$; ${ }^{13} \mathrm{C}-\mathrm{NMR}\left(75.0 \mathrm{MHz}, \mathrm{CDCl}_{3}\right) \delta 214.9,159.0,130.7,128.6,113.8,84.9,65.1,61.7$, 58.4, 55.8, 55.3, 36.8, 31.4, 29.2, 25.7, 23.1, 20.0, 7.91; HRMS (ESI ${ }^{+}$, TFA-Na) calcd for $\mathrm{C}_{20} \mathrm{H}_{30} \mathrm{NaO}_{5} 373.1991[\mathrm{M}+\mathrm{Na}]^{+}$, found $m / z$ 373.1975.

(3S,9R)-9-((4-Methoxybenzyl)oxy)-9-methyl-8-oxoundec-1-en-3-yl acrylate (11). To a solution of $2(195 \mathrm{mg}, 0.56 \mathrm{mmol})$ in THF:MeCN (4:1, 5.6 ml) were added imidazole $(227 \mathrm{mg}, 3.33 \mathrm{mmol}), \mathrm{PPh}_{3}(437 \mathrm{mg}, 1.67 \mathrm{mmol})$ and $\mathrm{I}_{2}(423 \mathrm{mg}$, $1.67 \mathrm{mmol}$ ) at $0^{\circ} \mathrm{C}$ under $\mathrm{N}_{2}$. After warming to $\mathrm{rt}$, the reaction mixture was stirred for $1 \mathrm{~h}$. The reaction was quenched with $\mathrm{H}_{2} \mathrm{O}$ and the aqueous phase was extracted with $\mathrm{CH}_{2} \mathrm{Cl}_{2}$. The combined organic extracts were dried over anhydrous $\mathrm{Na}_{2} \mathrm{SO}_{4}$ and concentrated in vacuo. The residue was purified by flash column chromatography (30:1 hexanes/EtOAc) to give the corresponding iodide $(204 \mathrm{mg}, 80 \%)$ as a colorless oil.

$[\alpha]_{\mathrm{D}}^{25}+6.68$ (c $\left.1.0 \mathrm{CHCl}_{3}\right)$; IR (KBr) 2936, 2864, 1712, 1613, 1514, $1460 \mathrm{~cm}^{-1} ;{ }^{1} \mathrm{H}-\mathrm{NMR}\left(300 \mathrm{MHz}, \mathrm{CDCl}_{3}\right) \delta 7.28(\mathrm{~d}, J=8.8 \mathrm{~Hz}, 2 \mathrm{H}), 6.90$ (d, $J=8.8 \mathrm{~Hz}, 2 \mathrm{H}), 4.33(\mathrm{~d}, J=10.7 \mathrm{~Hz}, 1 \mathrm{H}), 4.30(\mathrm{~d}, J=10.7 \mathrm{~Hz}, 1 \mathrm{H}), 3.80(\mathrm{~s}$, $3 \mathrm{H}), 3.26-3.21(\mathrm{~m}, 1 \mathrm{H}), 3.05-3.00(\mathrm{~m}, 1 \mathrm{H}), 2.97(\mathrm{ddd}, J=4.7,2.3 \mathrm{~Hz}, 1 \mathrm{H}), 2.79$ (ddd, $J=5.6,5.5,1.9 \mathrm{~Hz}, 1 \mathrm{H}), 2.66(\mathrm{dt}, J=7.0,3.8 \mathrm{~Hz}, 2 \mathrm{H}), 1.88-1.66(\mathrm{~m}, 2 \mathrm{H})$, $1.64-1.52(\mathrm{~m}, 4 \mathrm{H}), 1.48-1.40(\mathrm{~m}, 2 \mathrm{H}), 1.33(\mathrm{~s}, 3 \mathrm{H}), 0.84(\mathrm{t}, J=7.5 \mathrm{~Hz}, 3 \mathrm{H})$; ${ }^{13} \mathrm{C}-\mathrm{NMR}\left(75.0 \mathrm{MHz}, \mathrm{CDCl}_{3}\right) \delta 214.7,159.1,130.7,128.6,113.9,84.9,65.1$, 62.4, 58.3, 36.8, 31.6, 29.2, 25.6, 23.2, 20.0, 7.93, 5.02; HRMS (ESI ${ }^{+}$, TFA-Na) calcd for $\mathrm{C}_{20} \mathrm{H}_{29} \mathrm{INaO}_{4} 483.1008[\mathrm{M}+\mathrm{Na}]^{+}$, found $\mathrm{m} / z$ 483.1012.

To a solution of the iodide $(163 \mathrm{mg}, 0.35 \mathrm{mmol})$ in $\mathrm{MeOH}(707 \mu \mathrm{l})$ were added $\mathrm{NaI}(133 \mathrm{mg}, 0.88 \mathrm{mmol})$ and $\mathrm{Zn}(69.4 \mathrm{mg}, 1.06 \mathrm{mmol})$ under $\mathrm{N}_{2}$. After stirring for $2.5 \mathrm{~h}$ at $90^{\circ} \mathrm{C}$, the reaction was quenched with $\mathrm{H}_{2} \mathrm{O}$ and the aqueous phase was extracted with $\mathrm{CH}_{2} \mathrm{Cl}_{2}$. The combined organic extracts were dried over anhydrous $\mathrm{Na}_{2} \mathrm{SO}_{4}$ and concentrated in vacuo. The residue was purified by flash column chromatography (10:1 hexanes/EtOAc) to afford the corresponding allyl alcohol (118 mg, quant.) as a colorless oil.

$[\alpha]_{\mathrm{D}}^{26}+8.93\left(c \quad 1.0 \quad \mathrm{CHCl}_{3}\right)$; IR (KBr) 3518, 3075, 2941, 1710, 1613, $1513 \mathrm{~cm}^{-1} ;{ }^{1} \mathrm{H}-\mathrm{NMR}\left(300 \mathrm{MHz}, \mathrm{CDCl}_{3}\right) \delta 7.28(\mathrm{~d}, J=8.8 \mathrm{~Hz}, 2 \mathrm{H}), 6.89$ $(\mathrm{d}, J=8.8 \mathrm{~Hz}, 2 \mathrm{H}), 5.90-5.79(\mathrm{~m}, 1 \mathrm{H}), 5.20(\mathrm{ddd}, J=17.2,1.4 \mathrm{~Hz}, 1 \mathrm{H}), 5.09$ (ddd, $J=10.4,1.4 \mathrm{~Hz}, 1 \mathrm{H}), 4.32(\mathrm{~d}, J=10.8 \mathrm{~Hz}, 1 \mathrm{H}), 4.29(\mathrm{~d}, J=10.8 \mathrm{~Hz}, 1 \mathrm{H})$, $4.12-4.05(\mathrm{~m}, 1 \mathrm{H}) 3.80(\mathrm{~s}, 3 \mathrm{H}), 2.65(\mathrm{dt}, J=7.3,4.3 \mathrm{~Hz}, 2 \mathrm{H}), 1.87-1.68$ $(\mathrm{m}, 2 \mathrm{H}), 1.61-1.34(\mathrm{~m}, 6 \mathrm{H}), 1.32(\mathrm{~s}, 3 \mathrm{H}), 0.84(\mathrm{t}, J=7.5 \mathrm{~Hz}, 3 \mathrm{H}) ;{ }^{13} \mathrm{C}-\mathrm{NMR}$ $\left(75.0 \mathrm{MHz}, \mathrm{CDCl}_{3}\right) \delta 215.0,159.0,141.1,130.7,128.6,128.6,114.5,113.8$, $113.8,84.8,72.9,65.1,55.2,36.8,36.8,29.2,25.0,23.2,20.0,7.84$; HRMS (ESI ${ }^{+}$, TFA-Na) calcd for $\mathrm{C}_{20} \mathrm{H}_{30} \mathrm{NaO}_{4} 357.2042[\mathrm{M}+\mathrm{Na}]^{+}$, found $\mathrm{m} / z$ z 357.2025.

To a solution of the allyl alcohol $(179 \mathrm{mg}, 0.53 \mathrm{mmol})$ in $\mathrm{CH}_{2} \mathrm{Cl}_{2}(5.3 \mathrm{ml})$ were added acryloyl chloride $(65 \mu \mathrm{l}, 0.80 \mathrm{mmol}), \mathrm{Et}_{3} \mathrm{~N}(223 \mu \mathrm{l}, 1.60 \mathrm{mmol})$ and DMAP (cat.) at $0{ }^{\circ} \mathrm{C}$ under $\mathrm{N}_{2}$. After stirring for $1 \mathrm{~h}$ at $\mathrm{rt}$, the reaction was 
quenched with $\mathrm{H}_{2} \mathrm{O}$ and the aqueous phase was extracted with $\mathrm{CH}_{2} \mathrm{Cl}_{2}$. The combined organic extracts were dried over anhydrous $\mathrm{Na}_{2} \mathrm{SO}_{4}$ and concentrated in vacuo. The residue was purified by flash column chromatography (30:1 hexanes/EtOAc) to afford $\mathbf{1 1}(187 \mathrm{mg}, 90 \%)$ as a colorless oil.

$[\alpha]_{\mathrm{D}}^{24}+6.32\left(c 1.0 \mathrm{CHCl}_{3}\right)$; IR $(\mathrm{KBr}) 2940,1718,1615,1514,1460 \mathrm{~cm}^{-1}$; ${ }^{1} \mathrm{H}-\mathrm{NMR}\left(300 \mathrm{MHz}, \mathrm{CDCl}_{3}\right) \delta 7.27(\mathrm{~d}, J=8.8 \mathrm{~Hz}, 2 \mathrm{H}), 6.89(\mathrm{~d}, J=8.8 \mathrm{~Hz}, 2 \mathrm{H})$, $6.40(\mathrm{dd}, J=17.3,1.5 \mathrm{~Hz}, 1 \mathrm{H}), 6.12(\mathrm{dd}, J=17.3,10.4 \mathrm{~Hz}, 1 \mathrm{H}), 5.84-5.73$ $(\mathrm{m}, 2 \mathrm{H}), 5.31-5.27(\mathrm{~m}, 1 \mathrm{H}), 5.24(\mathrm{ddd}, J=17.3,9.3,1.3 \mathrm{~Hz}, 1 \mathrm{H}), 5.16$ (ddd, $J=10.5,5.9,1.2 \mathrm{~Hz}, 1 \mathrm{H}), 4.32(\mathrm{~d}, J=10.7 \mathrm{~Hz}, 1 \mathrm{H}), 4.29(\mathrm{~d}, J=10.7 \mathrm{~Hz}, 1 \mathrm{H}), 3.81$ (s, $3 \mathrm{H}), 2.64(\mathrm{dt}, J=7.3,3.9 \mathrm{~Hz}, 2 \mathrm{H}), 1.87-1.53(\mathrm{~m}, 8 \mathrm{H}), 1.32(\mathrm{~s}, 3 \mathrm{H}), 0.84$ $(\mathrm{t}, J=7.5 \mathrm{~Hz}, 3 \mathrm{H}) ;{ }^{13} \mathrm{C}-\mathrm{NMR}\left(75.0 \mathrm{MHz}, \mathrm{CDCl}_{3}\right) \delta 214.8,165.5,159.0,136.3$, 130.7, 130.6, 128.7, 128.6, 116.8, 113.8, 113.8, 84.5, 74.8, 65.1, 55.3, 36.8, 34.1, 29.2, 24.8, 23.3, 20.0, 7.90; HRMS (ESI ${ }^{+}$, TFA-Na) calcd for $\mathrm{C}_{23} \mathrm{H}_{32} \mathrm{NaO}_{5}$ $411.2147[\mathrm{M}+\mathrm{Na}]^{+}$, found $\mathrm{m} / z$ 411.2136.

(4S,10R)-avenolide (1). To a solution of $11(187 \mathrm{mg}, 0.48 \mathrm{mmol})$ in $\mathrm{CH}_{2} \mathrm{Cl}_{2}: \mathrm{H}_{2} \mathrm{O}(20: 1,4.6 \mathrm{ml})$ was added DDQ $(146 \mathrm{mg}, 0.53 \mathrm{mmol})$ at $\mathrm{rt}$ under $\mathrm{N}_{2}$. After stirring for $1 \mathrm{~h}$ at $\mathrm{rt}$, the reaction was quenched with $\mathrm{H}_{2} \mathrm{O}$ and the aqueous phase was extracted with $\mathrm{CH}_{2} \mathrm{Cl}_{2}$. The combined organic extracts were dried over anhydrous $\mathrm{Na}_{2} \mathrm{SO}_{4}$ and concentrated in vacuo. The residue was purified by flash column chromatography (40:1 hexanes/EtOAc) to give the corresponding alcohol (159 mg, quant.) as a colorless oil.

$[\alpha]_{\mathrm{D}}^{25}-8.51$ (c $\left.1.0 \mathrm{CHCl}_{3}\right)$; IR (KBr) 3486, 2936, 2863, 1721, 1637, $1459 \mathrm{~cm}^{-1} ;{ }^{1} \mathrm{H}-\mathrm{NMR}\left(300 \mathrm{MHz}, \mathrm{CDCl}_{3}\right) \delta 6.41(\mathrm{dd}, J=17.3,1.5 \mathrm{~Hz}, 1 \mathrm{H})$, $6.12(\mathrm{dd}, J=17.3,10.4 \mathrm{~Hz}, 1 \mathrm{H}), 5.85-5.73(\mathrm{~m}, 2 \mathrm{H}), 5.34-5.16(\mathrm{~m}, 2 \mathrm{H}), 3.82$ (brs, $1 \mathrm{H}), 2.54-2.45(\mathrm{~m}, 2 \mathrm{H}), 1.75-1.59(\mathrm{~m}, 8 \mathrm{H}), 1.33(\mathrm{~s}, 3 \mathrm{H}), 0.79$ $(\mathrm{t}, J=7.4 \mathrm{~Hz}, 3 \mathrm{H}) ;{ }^{13} \mathrm{C}-\mathrm{NMR}\left(75.0 \mathrm{MHz}, \mathrm{CDCl}_{3}\right) \delta 214.3,165.5,136.2,130.8$, 128.6, 116.9, 78.9, 74.6, 35.5, 34.0, 32.3, 25.1, 24.7, 23.3, 7.65; HRMS (ESI ${ }^{+}$, TFA-Na) calcd for $\mathrm{C}_{15} \mathrm{H}_{24} \mathrm{NaO}_{4} 291.1572[\mathrm{M}+\mathrm{Na}]^{+}$, found $\mathrm{m} / \mathrm{z} 291.1576$.

To a solution of the alcohol $(38.1 \mathrm{mg}, 0.14 \mathrm{mmol})$ in $\mathrm{CH}_{2} \mathrm{Cl}_{2}(5.0 \mathrm{ml})$ was added a solution of Grubbs second-generation catalyst $(6.0 \mathrm{mg}, 0.01 \mathrm{mmol})$ in $\mathrm{CH}_{2} \mathrm{Cl}_{2}(9.2 \mathrm{ml})$ at $\mathrm{rt}$ under $\mathrm{N}_{2}$. The reaction mixture was stirred for $2 \mathrm{~h}$ at $40{ }^{\circ} \mathrm{C}$. After the reaction was complete, Quadrasil AP $(500 \mathrm{mg})$ was added to the reaction mixture. The suspension was stirred for $5 \mathrm{~min}$ at $\mathrm{rt}$ and then allowed to stand for $10 \mathrm{~min}$. The mixture was filtered through a pad of celite and the filtrate was washed with $\mathrm{H}_{2} \mathrm{O}$. The organic layers were dried over anhydrous $\mathrm{Na}_{2} \mathrm{SO}_{4}$ and concentrated in vacuo. The residue was purified by flash column chromatography (5:1 hexanes/EtOAc) to afford $\mathbf{1}(43.2 \mathrm{mg}$, quant.) as a colorless oil.

$[\alpha]_{\mathrm{D}}^{26}+2.27\left(c 1.0 \mathrm{CHCl}_{3}\right)$; IR (KBr) 3480, 2929, 2858, 1746, 1710, $1459 \mathrm{~cm}^{-1}$; ${ }^{1} \mathrm{H}-\mathrm{NMR}\left(300 \mathrm{MHz}, \mathrm{CDCl}_{3}\right) \delta 7.44(\mathrm{dd}, J=5.8,1.5 \mathrm{~Hz}, 1 \mathrm{H}), 6.11(\mathrm{dd}, J=5.7$, $2.0 \mathrm{~Hz}, 1 \mathrm{H}), 5.05-5.02(\mathrm{~m}, 1 \mathrm{H}), 3.76$ (brs, $1 \mathrm{H}), 2.56-2.47(\mathrm{~m}, 2 \mathrm{H}), 1.83-1.61(\mathrm{~m}$, $6 \mathrm{H}), 1.61-1.42(\mathrm{~m}, 2 \mathrm{H}), 1.33(\mathrm{~s}, 3 \mathrm{H}), 0.79(\mathrm{t}, J=7.4 \mathrm{~Hz}, 3 \mathrm{H}) ;{ }^{13} \mathrm{C}-\mathrm{NMR}(75.0 \mathrm{MHz}$, $\left.\mathrm{CDCl}_{3}\right) \delta 214.2,173.0,156.1,121.7,83.0,78.9,35.4,33.0,32.4,25.2,24.6,23.2,7.66$; HRMS (FAB, $m$-NBA) calcd for $\mathrm{C}_{13} \mathrm{H}_{21} \mathrm{O}_{4} 241.1440[\mathrm{M}+\mathrm{H}]^{+}$, found $m / z$ 241.1447.

(4S,10S)-avenolide (19). $[\alpha]_{\mathrm{D}}^{24}+48.91\left(c 1.0, \mathrm{CHCl}_{3}\right)$; IR (KBr) 3483, 2940, 2874, $1748,1711,1459 \mathrm{~cm}^{-1} ;{ }^{1} \mathrm{H}-\mathrm{NMR}\left(300 \mathrm{MHz}, \mathrm{CDCl}_{3}\right) \delta 7.45(\mathrm{dd}, J=5.7,1.5 \mathrm{~Hz}$, $1 \mathrm{H}), 6.12(\mathrm{dd}, J=5.7,2.1 \mathrm{~Hz}, 1 \mathrm{H}), 5.08-5.03(\mathrm{~m}, 1 \mathrm{H}), 3.77$ (brs, $1 \mathrm{H}), 2.60-2.45(\mathrm{~m}$, $2 \mathrm{H}), 1.87-1.41(\mathrm{~m}, 8 \mathrm{H}), 1.34(\mathrm{~s}, 3 \mathrm{H}), 0.81(\mathrm{t}, J=7.5 \mathrm{~Hz}, 3 \mathrm{H}) ;{ }^{13} \mathrm{C}-\mathrm{NMR}(75.0 \mathrm{MHz}$, $\left.\mathrm{CDCl}_{3}\right) \delta 214.1,173.0,156.0,121.7,83.0,78.9,35.4,33.0,32.4,25.2,24.6,23.1,7.66$; HRMS (FAB, $m$-NBA) calcd for $\mathrm{C}_{13} \mathrm{H}_{21} \mathrm{O}_{4} 241.1440[\mathrm{M}+\mathrm{H}]^{+}$, found $m / z$ 241.1438.

(4R,10R)-avenolide (22). $\quad[\alpha]_{\mathrm{D}}^{26}-51.07\left(c 1.0 . \mathrm{CHCl}_{3}\right) ; \mathrm{IR}(\mathrm{KBr}) 3482,3093$, 2930, 1748, $1458 \mathrm{~cm}^{-1}$; ${ }^{1} \mathrm{H}-\mathrm{NMR}\left(300 \mathrm{MHz}, \mathrm{CDCl}_{3}\right) \delta 7.45(\mathrm{dd}, J=5.7,1.5 \mathrm{~Hz}$, $1 \mathrm{H}), 6.12(\mathrm{dd}, J=5.7,2.1 \mathrm{~Hz}, 1 \mathrm{H}), 5.07-5.02(\mathrm{~m}, 1 \mathrm{H}), 3.76$ (brs, $1 \mathrm{H}), 2.59-2.45$ $(\mathrm{m}, 2 \mathrm{H}), 1.86-1.34(\mathrm{~m}, 8 \mathrm{H}), 1.34(\mathrm{~s}, 3 \mathrm{H}), 0.80(\mathrm{t}, J=7.4 \mathrm{~Hz}, 3 \mathrm{H}) ;{ }^{13} \mathrm{C}-\mathrm{NMR}$ $\left(75.0 \mathrm{MHz}, \mathrm{CDCl}_{3}\right) \delta 214.4,173.2,156.2,121.9,83.2,79.1,35.6,33.2,32.6$, 35.4, 24.9, 23.3, 7.86; HRMS (ESI ${ }^{+}$, TFA-Na) calcd for $\mathrm{C}_{13} \mathrm{H}_{20} \mathrm{NaO}_{4} 263.1259$ $[\mathrm{M}+\mathrm{Na}]^{+}$, found $\mathrm{m} / \mathrm{z} 263.1271$

10-Deoxy avenolide (28). $[\alpha]_{\mathrm{D}}^{25}+47.75\left(\right.$ c 1.0. $\left.\mathrm{CHCl}_{3}\right)$; IR ( $\left.\mathrm{KBr}\right) 3503,3091$, 2930, $1753,1708 \mathrm{~cm}^{-1}$; ${ }^{1} \mathrm{H}-\mathrm{NMR}\left(300 \mathrm{MHz}, \mathrm{CDCl}_{3}\right) \delta 7.45(\mathrm{dd}, J=5.7,1.5 \mathrm{~Hz}$, $1 \mathrm{H}), 6.11(\mathrm{dd}, J=5.7,2.1 \mathrm{~Hz}, 1 \mathrm{H}), 5.07-5.02(\mathrm{~m}, 1 \mathrm{H}), 2.47-2.40(\mathrm{~m}, 3 \mathrm{H})$, $1.81-1.28(\mathrm{~m}, 8 \mathrm{H}), 1.05(\mathrm{~d}, J=7.0 \mathrm{~Hz}, 3 \mathrm{H}), 0.87(\mathrm{t}, J=7.5 \mathrm{~Hz}, 3 \mathrm{H}) ;{ }^{13} \mathrm{C}-\mathrm{NMR}$ $\left(75.0 \mathrm{MHz}, \mathrm{CDCl}_{3}\right) \delta 214.4,173.1,156.2,121.6,83.1,47.9,40.6,33.1,25.9$,
24.7, 23.1, 15.9, 11.7; HRMS (ESI ${ }^{+}$, TFA-Na) calcd for $\mathrm{C}_{13} \mathrm{H}_{20} \mathrm{NaO}_{3} 247.1310$ $[\mathrm{M}+\mathrm{Na}]^{+}$, found $\mathrm{m} / z 247.1322$.

\section{Evaluation of biological activity}

Biological activity of the compounds were measured either as the DNA dissociation activity from the rAvaR1-DNA complex in gel-shift experiment or by avermectin-inducing activity of $S$. avermitilis aco mutant. ${ }^{6}$

\section{ACKNOWLEDGEMENTS}

This work was supported by a Grant-in-Aid for Scientific Research on Innovative Areas from MEXT Japan (HI), from JSPS 20310122 (HI) and from the Institute for Fermentation, Osaka, Japan (HI). We thank N Sato and K Nagai of Kitasato University for measuring NMR and MS spectra.

1 Horinouchi, S. \& Beppu, T. Autoregulators. in Genetics and Biochemistry of Antibiotic Production (ed. Vining, L.) 103-119 (Butterworth-Heinemann, Newton, MA, 1994).

2 Yamada, Y. Autoregulatory factors and regulation of antibiotic production in Streptomyces. In: Microbial Signalling and Communication (eds. England, R., Hobbs, G., Bainton, N. \& Roberts, D.McL.) 177-196 (Society for General Microbiology, Cambridge, 1999).

3 Kondo, K., Higuchi, Y., Sakuda, S., Nihira, T. \& Yamada, Y. New viriginiae butanolides from Streptomyces virginiae. J. Antibiot. 42, 769-778 (1989).

4 Sato, K., Nihira, T., Sakuda, S., Yanagimoto, M. \& Yamada, Y. Isolation and structure of a new butyrolactone autoregulator from Streptomyces sp. FRI-5. J. Ferment. Bioeng. 68, 170-173 (1989).

5 Takano, E. et al. Purification and structural determination of SCB1, a $\gamma$-butyrolactone that elicits antibiotic production in Streptomyces coelicolor A3 (2). J. Biol. Chem. 275, 11010-11016 (2000)

6 Kitani, S. et al. Avenolide, a novel Streptomyces hormone controlling antibiotic production in Streptomyces avermitilis. Proc. Natl. Acad. Sci. USA 108, 1641016415 (2011)

7 Mukku, V. J. R., Speitling, M., Laatsch, H. \& Helmke, E. New butenolides from two marine streptomycetes. J. Nat. Prod. 63, 1570-1572 (2000).

8 Gawronski, J. K., van Oeveren, A., van der Deen, H., Leung, C. W. J. \& Feringa, B. L. Simple circular dichroic method for the determination of absolute configuration of 5-substituted 2(5H)-furanones. J. Org. Chem. 61, 1513-1515 (1996).

9 Jacobsen, E. N., Marko, I., Mungall, W. S., Schroder, G. \& Sharpless, K. B. Asymmetric dihydroxylation via ligand-accelerated catalysis. J. Am. Chem. Soc. 110, 1968-1970 (1988).

10 Kolb, H., VanNiewenhze, M. S. \& Sharpless, K. B. Catalytic asymmetric dihydroxylation. Chem Rev 94, 2483-2547 (1994).

11 Corey, E. J., Guzman-Perez, A. \& Noe, M. C. The application of a mechanistic model leads to the extension of the Sharpless asymmetric dihydroxylation to allylic 4-methoxybenzoates and conformationally related amine and homoallylic alcohol derivatives. J. Am. Chem. Soc. 117, 10805-10816 (1995).

12 Nagamitsu, T. et al. Total synthesis of nafuredin- $\gamma$, a $\gamma$-lactone related to nafuredin with selective inhibitory activity against $\mathrm{NADH}$-fumarate reductase. Tetrahedron Lett. 44, 6441-6444 (2003).

13 Nagamitsu, T. et al. The total synthesis and biological evaluation of nafuredin- $\gamma$ and its analogues. Tetrahedron 64, 8117-8127 (2008).

14 Parikh, J. R. \& Doering, W.v.E. Sulfur trioxide in the oxidation of alcohols by dimethyl sulfoxide. J. Am. Chem. Soc. 89, 5505-5507 (1967).

15 Liu, L. \& Floreancig, P. E. Cyclization reactions through DDQ-mediated vinyl oxazolidinone oxidation. Org. Lett. 11, 3152-3155 (2009).

16 Liu, J.- H., Song, L.- D. \& Long, Y.- Q. Studies toward the total synthesis of cyclodidemniserinol trisulfate. Part I: 3,5,7-Trisubstituted 6,8-dioxabicyclo [3.2.1] octane core structure construction via a convergent and a linear stereoselective synthesis. Tetrahedron Lett. 50, 4587-4591 (2009).

17 Katsuki, T. \& Sharpless, K. B. The first practical method for asymmetric epoxidation. J. Am. Chem. Soc. 102, 5974-5976 (1980)

18 Trnka, T. M. \& Grubbs, R. H. The development of $\mathrm{L}_{2} \mathrm{X}_{2} \mathrm{Ru}=\mathrm{CHR}$ olefin metathesis catalysts: an organometallic success story. Acc. Chem. Res. 34, 18-29 (2001).

19 Fürstner, A. Olefin metathesis and beyond. Angew. Chem. Int. Ed. Engl. 39, 3012-3043 (2000).

20 Grubbs, R. H. \& Chang, S. Recent advances in olefin metathesis and its application in organic synthesis. Tetrahedron 54, 4413-4450 (1998).

21 Schuster, M. \& Blechert, S. Olefin metathesis in organic chemistry. Angew. Chem. Int. Ed. Engl. 36, 2036-2056 (1997).

22 Fürstner, A. Recent advancements in ring closing olefin metathesis. Top. Catal. 4, 285-299 (1997).

23 Seebach, D. \& Steinmueller, D. Preparation of (S)-2-methylbutyl and (S)-sec-butyl ketones from optically active 2-methyl-1-butanol by the dithiane method. Angew. Chem. Int. Ed. Engl. 7, 619-620 (1968). 